\title{
Description of atmospheric conditions at the Pierre Auger Observatory using the Global Data Assimilation System (GDAS)
}

The Pierre Auger Collaboration, P. Abreu ${ }^{\text {bk }}$, M. Aglietta ${ }^{\text {ay }}$, M. Ahlers ${ }^{\text {co }}$, E.J. Ahn ${ }^{c c}$, I.F.M. Albuquerque ${ }^{\text {, }}$, D. Allard ${ }^{\mathrm{ab}}$, I. Allekotte ${ }^{\mathrm{a}}$, J. Allen ${ }^{\mathrm{cg}}$, P. Allison ${ }^{\mathrm{ci}}$, A. Almela ${ }^{\mathrm{k}, \mathrm{g}}$, J. Alvarez Castillo ${ }^{\mathrm{bd}}$, J. Alvarez-Muñiz ${ }^{\mathrm{bu}}$, M. Ambrosio as, A. Aminaei ${ }^{\text {be }}$, L. Anchordoqui ${ }^{\text {cp }}$, S. Andringa bk, T. Antiči'c w, C. Aramo as, E. Arganda d,br F. Arqueros ${ }^{\text {br }}$, H. Asorey ${ }^{a}$, P. Assis ${ }^{\text {bk }}$, J. Aublin ${ }^{\text {ad }}$, M. Ave ${ }^{a j}$, M. Avenier ${ }^{\text {ae }}$, G. Avila $^{j}$, T. Bäcker $^{\text {an }}$, A.M. Badescu ${ }^{\text {bn }}$, M. Balzer ${ }^{\text {ai }}$, K.B. Barber ${ }^{1}$, A.F. Barbosa ${ }^{\mathrm{m}}$, R. Bardenet ${ }^{\text {ac }}$, S.L.C. Barroso ${ }^{\text {, }}$, B. Baughman ${ }^{\text {ci, } 1}$, J. Bäuml $^{\text {ah }}$, J.J. Beatty ${ }^{\text {ci }}$, B.R. Becker ${ }^{\text {cn }}$, K.H. Becker ${ }^{\text {ag }}$, A. Bellétoile af, J.A. Bellido ${ }^{1}$, S. BenZvi ${ }^{\text {co }}$, C. Berat $^{\text {ae }}$, X. Bertou ${ }^{\text {a }}$, P.L. Biermann ${ }^{\text {ak }}$, P. Billoir ${ }^{\text {ad }}$, F. Blanco $^{\text {br }}$, M. Blanco ${ }^{\text {ad,bs }}$, C. Bleve $^{\text {ag }}$, H. Blümer $^{\text {aj,ah }}$, M. Boháčová ${ }^{\mathrm{y}}$, D. Boncioli ${ }^{\text {at }}$, C. Bonifazi ${ }^{\text {u,ad }}$, R. Bonino ay, N. Borodai ${ }^{\text {bi }}$, J. Brack ${ }^{\text {ca }}$, I. Brancus ${ }^{\text {bl }}$, P. Brogueira ${ }^{\text {bk }}$, W.C. Brown ${ }^{\text {cb }}$, R. Bruijn ${ }^{\text {bw, }}{ }^{\text {, P. Buchholz }}{ }^{\text {an }}$, A. Bueno ${ }^{\text {bt }}$, R.E. Burton ${ }^{\text {by }}$, K.S. Caballero-Mora ${ }^{\text {cj }}$, B. Caccianiga ${ }^{\text {aq }}$, L. Caramete $^{\text {ak }}$, R. Caruso ${ }^{\text {au }}$, A. Castellina ${ }^{\text {ay }}$, O. Catalano ${ }^{a x}$, G. Cataldi $^{\text {ar }}$, L. Cazon ${ }^{\text {bk }}$, R. Cester ${ }^{\text {av }}$, J. Chauvin ${ }^{\text {ae }}$, S.H. Cheng ${ }^{\text {cj }}$, A. Chiavassa ${ }^{\text {ay }}$, J.A. Chinellato ${ }^{\mathrm{p}}$, J. Chirinos Diaz ${ }^{\text {cf }}$, J. Chudoba ${ }^{\mathrm{y}}$, R.W. Clay ${ }^{1}$, M.R. Coluccia ${ }^{\mathrm{ar}}$, R. Conceição ${ }^{\mathrm{bk}}$, F. Contreras ${ }^{i}$, H. Cook ${ }^{\text {bw }}$, M.J. Cooper ${ }^{1}$, J. Coppens ${ }^{\text {be,bg }}$, A. Cordier ${ }^{\text {ac }}$, S. Coutu ${ }^{\text {cj }}$, C.E. Covault ${ }^{\text {by }}$, A. Creusot ${ }^{\text {ab }}$, A. Criss ${ }^{\mathrm{cj}}$, J. Cronin ${ }^{\mathrm{cl}}$, A. Curutiu ${ }^{\mathrm{ak}}, \mathrm{S}$. Dagoret-Campagne ${ }^{\mathrm{ac}}, \mathrm{R}_{\text {. Dallier }}{ }^{\text {af }}$, B. Daniel ${ }^{\mathrm{p}}$, S. Dasso $^{\mathrm{e}, \mathrm{c}}$, K. Daumiller ${ }^{\text {ah }}$, B.R. Dawson ${ }^{\text {}}$, R.M. de Almeida ${ }^{v}$, M. De Domenico ${ }^{\text {au }}$, C. De Donato ${ }^{\text {bd }}$, S.J. de Jong ${ }^{\text {be,bg }}$,

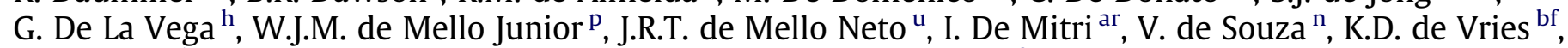
L. del Peral ${ }^{\text {bs }}$, M. del Río ${ }^{\text {at,i }}$, O. Deligny ${ }^{\text {aa }}$, H. Dembinski ${ }^{\text {aj }}$, N. Dhital ${ }^{\text {cf }}$, C. Di Giulio ${ }^{\text {at,ap }}$, M.L. Díaz Castro ${ }^{\mathrm{m}}$,

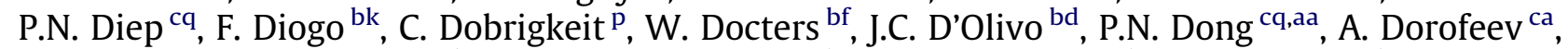
J.C. dos Anjos ${ }^{\mathrm{m}}$, M.T. Dova ${ }^{\mathrm{d}}$, D. D’Urso ${ }^{\mathrm{as}}$, I. Dutan ${ }^{\mathrm{ak}}$, J. Ebr $^{\mathrm{y}}$, R. Engel $^{\text {ah }}$, M. Erdmann $^{\mathrm{al}}$, C.O. Escobar ${ }^{\mathrm{cc}, \mathrm{p}}$, J. Espadanal ${ }^{\text {bk }}$, A. Etchegoyen ${ }^{\text {g,k }}$, P. Facal San Luis ${ }^{c l}$, I. Fajardo Tapia ${ }^{\text {bd }}$, H. Falcke $^{\text {be,bh }}$, G. Farrar $^{\text {cg }}$, A.C. Fauth ${ }^{\mathrm{p}}$, N. Fazzini ${ }^{\mathrm{cc}}$, A.P. Ferguson ${ }^{\text {by }}$, B. Fick ${ }^{\mathrm{cf}}$, A. Filevich ${ }^{\mathrm{g}}$, A. Filipčič $^{\text {bo,bp }}$, S. Fliescher ${ }^{\text {al }}$, C.E. Fracchiolla ${ }^{\text {ca }}$, E.D. Fraenkel ${ }^{\text {bf }}$, O. Fratu ${ }^{\text {bn }}$, U. Fröhlich ${ }^{\text {an }}$, B. Fuchs ${ }^{\text {aj }}$, R. Gaior ${ }^{\text {ad }}$, R.F. Gamarra ${ }^{g}$, S. Gambetta ${ }^{\text {ao }}$, B. García ${ }^{\text {h, S.T. Garcia Roca }}{ }^{\text {bu }}$, D. Garcia-Gamez ${ }^{\text {ac }}$, D. Garcia-Pinto ${ }^{\text {br }}$, A. Gascon ${ }^{\text {bt }}$, H. Gemmeke ${ }^{\text {ai }}$, P.L. Ghia ${ }^{\text {ad }}$, U. Giaccari ${ }^{\text {ar }}$, M. Giller ${ }^{\text {bj }}$, H. Glass ${ }^{c c}$, M.S. Gold ${ }^{c n}$, G. Golup ${ }^{\text {a }}$, F. Gomez Albarracin $^{\mathrm{d}}$, M. Gómez Berisso ${ }^{\mathrm{a}}$, P.F. Gómez Vitale ${ }^{\mathrm{j}}$, P. Gonçalves ${ }^{\mathrm{bk}}$, D. Gonzalez ${ }^{\text {aj }}$, J.G. Gonzalez ${ }^{\text {ah }}$, B. Gookin ${ }^{\text {ca }}$, A. Gorgi ${ }^{\text {ay }}$, P. Gouffon ${ }^{\text {}}$, E. Grashorn ${ }^{\text {ci }}$, S. Grebe ${ }^{\text {be,bg }}$, N. Griffith ${ }^{\text {ci }}$, M. Grigat ${ }^{\text {al }}$, A.F. Grillo $^{\text {az }}$, Y. Guardincerri ${ }^{\text {c }}$, F. Guarino ${ }^{\text {as }}$, G.P. Guedes ${ }^{\text {q }}$, A. Guzman ${ }^{\text {bd }}$, P. Hansen ${ }^{\text {d }}$, D. Harari ${ }^{\text {a }}$, T.A. Harrison ${ }^{1}$, J.L. Harton ${ }^{\text {ca }}$, A. Haungs ${ }^{\text {ah }}$, T. Hebbeker ${ }^{\text {al }}$, D. Heck ${ }^{\text {ah }}$, A.E. Herve ${ }^{1}$, C. Hojvat ${ }^{\text {cc }}$, N. Hollon ${ }^{\text {cl }}$, V.C. Holmes ${ }^{1}$, P. Homola ${ }^{\text {bi }}$, J.R. Hörandel ${ }^{\text {be }}$, A. Horneffer ${ }^{\text {be }}$, P. Horvath ${ }^{z}$, M. Hrabovský ${ }^{z, y}$, D. Huber ${ }^{\text {aj }}$, T. Huege ${ }^{\text {ah }}$, A. Insolia $^{\text {au }}$, F. Ionita ${ }^{\mathrm{cl}}$, A. Italiano ${ }^{\text {au }}$, C. Jarne ${ }^{\mathrm{d}}$, S. Jiraskova ${ }^{\text {be }}$, M. Josebachuili ${ }^{\mathrm{g}}$, K. Kadija ${ }^{\text {w }}$, K.H. Kampert ${ }^{\text {ag }}$, P. Karhan ${ }^{\text {x }}$ P. Kasper ${ }^{\text {cc }}$, B. Kégl ac, B. Keilhauer ${ }^{\text {ah,* }}$, A. Keivani ${ }^{\text {ce }}$, J.L. Kelley ${ }^{\text {be }}$, E. Kemp ${ }^{\text {p }}$, R.M. Kieckhafer ${ }^{\text {cf }}$, H.O. Klages ${ }^{\text {ah }}$, M. Kleifges ${ }^{\text {ai }}$, J. Kleinfeller ${ }^{\text {i,ah }}$, J. Knapp ${ }^{\text {bw }}$, D.-H. Koang ${ }^{\text {ae }}$, K. Kotera ${ }^{\text {cl }}$, N. Krohm ${ }^{\text {ag }}$, O. Krömer ${ }^{\text {ai }}$, D. Kruppke-Hansen ${ }^{\text {ag }}$, F. Kuehn ${ }^{\text {cc }}$, D. Kuempel ${ }^{\text {an,ag }}$, J.K. Kulbartz ${ }^{\text {am }}$, N. Kunka ${ }^{\text {ai }}$, G. La Rosa ${ }^{\text {ax }}$,

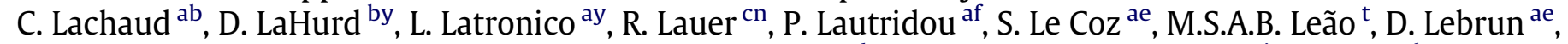
P. Lebrun ${ }^{c c}$, M.A. Leigui de Oliveira ${ }^{t}$, A. Letessier-Selvon ${ }^{\text {ad }}$, I. Lhenry-Yvon ${ }^{\text {aa }}$, K. Link ${ }^{\text {aj }}$, R. López ${ }^{\text {ba }}$, A. Lopez Agüera ${ }^{\text {bu }}$, K. Louedec ${ }^{\text {ae,ac }}$, J. Lozano Bahilo ${ }^{\text {bt }}$, L. Lu ${ }^{\text {bw }}$, A. Lucero ${ }^{g}$, M. Ludwig aj, H. Lyberis ${ }^{\text {u,aa }}$,

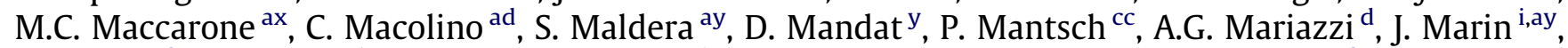
V. Marin ${ }^{\text {af }}$, I.C. Maris ad, H.R. Marquez Falcon ${ }^{\text {bc }}$, G. Marsella ${ }^{\text {aw }}$, D. Martello ${ }^{\text {ar }}$, L. Martin ${ }^{\text {af }}$, H. Martinez ${ }^{\text {bb }}$, O. Martínez Bravo ${ }^{\text {ba }}$, H.J. Mathes ${ }^{\text {ah }}$, J. Matthews ${ }^{\text {ce,ck, }}$, J.A.J. Matthews ${ }^{c n}$, G. Matthiae $^{\text {at }}$, D. Maurel ${ }^{\text {ah }}$, D. Maurizio $^{\text {av }}$, P.O. Mazur ${ }^{\text {cc }}$, G. Medina-Tanco ${ }^{\text {bd }}$, M. Melissas ${ }^{\text {aj }}$, D. Melo ${ }^{\mathrm{g}}$, E. Menichetti ${ }^{\text {av }}$, A. Menshikov ${ }^{\text {ai }}$, 
P. Mertsch ${ }^{\text {bv }}$, C. Meurer ${ }^{\text {al }}$, S. Mi'canovi'c ${ }^{w}$, M.I. Micheletti ${ }^{\text {f }}$ I.A. Minaya ${ }^{\text {br }}$, L. Miramonti ${ }^{\text {aq }}$, L. Molina-Bueno $^{\mathrm{bt}}, \mathrm{S}$. Mollerach ${ }^{\mathrm{a}}, \mathrm{M}$. Monasor ${ }^{\mathrm{cl}}$, D. Monnier Ragaigne ${ }^{\mathrm{ac}}, \mathrm{F}_{\text {. Montanet }}^{\mathrm{ae}}$, B. Morales ${ }^{\mathrm{bd}}$, C. Morello $^{\text {ay }}$, E. Moreno ${ }^{\text {ba }}$, J.C. Moreno ${ }^{\mathrm{d}}$, M. Mostafáca ${ }^{\text {, }}$ C.A. Moura ${ }^{\mathrm{t}}$, M.A. Muller ${ }^{\mathrm{p}}$, G. Müller $^{\text {al }}$, M. Münchmeyer ${ }^{\text {ad }}$, R. Mussa ${ }^{\text {av }}$, G. Navarra ${ }^{\text {ay, }}{ }^{3}$, J.L. Navarro $^{f}$, S. Navas ${ }^{\text {bt }}$, P. Necesal ${ }^{\mathrm{y}}$, L. Nellen ${ }^{\text {bd }}$, A. Nelles ${ }^{\text {be,bg }}{ }^{\text {J. }}$. Neuser ${ }^{\text {ag }}$, P.T. Nhung ${ }^{\text {cq }}$, M. Niechciol ${ }^{\text {an }}$, L. Niemietz $^{\text {ag }}$, N. Nierstenhoefer ${ }^{\text {ag }}$, D. Nitz $^{\text {cf }}$, D. Nosek $^{\mathrm{x}}$, L. Nožka ${ }^{\mathrm{y}}$, J. Oehlschläger ${ }^{\text {ah }}$, A. Olinto ${ }^{\mathrm{cl}}, \mathrm{M}$ Ortiz $^{\text {br }}$, N. Pacheco $^{\mathrm{bs}}$, D. Pakk Selmi-Dei $^{\mathrm{p}}$,

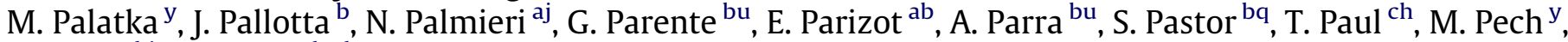
J. Pȩkala ${ }^{\text {bi }}$, R. Pelayo ${ }^{\text {ba,bu }}{ }^{\text {, I.M. Pepe }}{ }^{\mathrm{s}}$, L. Perrone ${ }^{\mathrm{aw}}$, R. Pesce ${ }^{\mathrm{ao}}$, E. Petermann ${ }^{\mathrm{cm}}$, S. Petrera ${ }^{\text {ap }}$, P. Petrinca ${ }^{\text {at }}$, A. Petrolini ${ }^{\text {ao }}$, Y. Petrov ${ }^{\text {ca }}$, C. Pfendner ${ }^{c o}$, R. Piegaia ${ }^{c}$, T. Pierog ${ }^{\text {ah }}$, P. Pieroni ${ }^{c}$, M. Pimenta ${ }^{\text {bk }}$, V. Pirronello ${ }^{\text {au }}$,

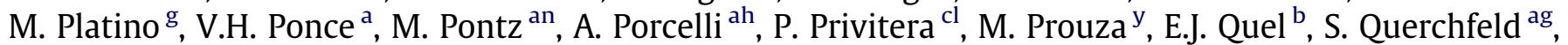
J. Rautenberg ${ }^{\text {ag }}$, O. Ravel ${ }^{\text {af }}$, D. Ravignani ${ }^{g}$, B. Revenu ${ }^{\text {af }}$, J. Ridky ${ }^{\mathrm{y}}$, S. Riggi ${ }^{\text {bu }}$, M. Risse ${ }^{\text {an }}$, P. Ristori $^{\text {b }}$, H. Rivera ${ }^{\text {aq }}$, V. Rizi ${ }^{\text {ap }}$, J. Roberts ${ }^{c g}$, W. Rodrigues de Carvalho ${ }^{\text {bu }}$, G. Rodriguez $^{\text {bu }}$, J. Rodriguez Martino ${ }^{\mathrm{i}}$, J. Rodriguez Rojo ${ }^{i}$, I. Rodriguez-Cabo ${ }^{\text {bu }}$, M.D. Rodríguez-Frías ${ }^{\text {bs }}$, G. Ros $^{\text {bs }}$, J. Rosado ${ }^{\text {br }}$, T. Rossler ${ }^{z}$, M. Roth $^{\text {ah }}$, B. Rouillé-d'Orfeuil ${ }^{\text {cl }}$, E. Roulet ${ }^{\text {a }}$, A.C. Rovero ${ }^{\text {e }}$, C. Rühle ${ }^{\text {ai }}$, A. Saftoiu ${ }^{\text {bl }}$, F. Salamida ${ }^{\text {aa }}$, H. Salazar $^{\text {ba }}$, F. Salesa Greus ${ }^{\text {ca }}$, G. Salina ${ }^{\text {at }}$, F. Sánchez ${ }^{g}$, C.E. Santo ${ }^{\text {bk }}$, E. Santos ${ }^{\text {bk }}$, E.M. Santos ${ }^{\text {u }}$, F. Sarazin ${ }^{\text {bz }}$, B. Sarkar ${ }^{\text {ag }}$, S. Sarkar ${ }^{\text {bv }}$, R. Sato ${ }^{\text {i }}$, N. Scharf ${ }^{\text {al }}$, V. Scherini ${ }^{\text {aq }}$, H. Schieler ${ }^{\text {ah }}$, P. Schiffer ${ }^{\text {am,al }}$, A. Schmidt ${ }^{\text {ai }}$, O. Scholten ${ }^{\text {bf }}$, H. Schoorlemmer ${ }^{\text {be,bg }}{ }^{\text {, J. Schovancova }}{ }^{y}$, P. Schovánek ${ }^{y}$, F. Schröder ${ }^{\text {ah }}$, S. Schulte ${ }^{\text {al }}$, D. Schuster ${ }^{\text {bz }}$, S.J. Sciutto ${ }^{\text {d }}$, M. Scuderi ${ }^{\text {au }}$, A. Segreto ${ }^{a x}$, M. Settimo ${ }^{\text {an }}$, A. Shadkam ${ }^{\text {ce }}$, R.C. Shellard ${ }^{\mathrm{m}}$, I. Sidelnik ${ }^{\mathrm{g}}$, G. Sigl ${ }^{\text {am }}$, H.H. Silva Lopez ${ }^{\text {bd }}$, O. Sima ${ }^{\text {bm }}$, A. 'Smiałkowski ${ }^{\text {bj }}$, R. Šmída ah, G.R. Snow ${ }^{\text {cm }}$, P. Sommers ${ }^{\text {cj }}$, J. Sorokin ${ }^{\text {l }}$, H. Spinka ${ }^{\text {bx,cc }}$, R. Squartini ${ }^{i}$, Y.N. Srivastava ${ }^{\text {ch }}$, S. Stanic ${ }^{\text {bp }}$, J. Stapleton ${ }^{\text {ci }}$, J. Stasielak ${ }^{\text {bi }}$, M. Stephan ${ }^{\text {al }}$, A. Stutz ${ }^{\text {ae }}$, F. Suarez $^{\text {g }}$, T. Suomijärvi ${ }^{\text {aa }}$, A.D. Supanitsky ${ }^{\text {e }}$, T. Šuša ${ }^{\text {w }}$, M.S. Sutherland ${ }^{\text {ce }}$, J. Swain ${ }^{\text {ch }}$, Z. Szadkowski $^{\text {bj }}$, M. Szuba ${ }^{\text {ah }}$, A. Tapia ${ }^{g}$, M. Tartare ${ }^{\text {ae }}$, O. Taşcău ${ }^{\text {ag }}$, C.G. Tavera Ruiz ${ }^{\text {bd }}$, R. Tcaciuc ${ }^{\text {an }}$, N.T. Thao ${ }^{\text {cq }}$, D. Thomas ${ }^{\text {ca }}$, J. Tiffenberg ${ }^{c}$, C. Timmermans ${ }^{\text {bg,be }}{ }^{\text {, W. Wkaczyk }}{ }^{\text {bj }}$, C.J. Todero Peixoto ${ }^{n}$, G. Toma ${ }^{\text {bl }}$, L. Tomankova $^{\mathrm{y}}$, B. Tomé ${ }^{\mathrm{bk}}$, A. Tonachini ${ }^{\text {av }}$, P. Travnicek ${ }^{\mathrm{y}}$, D.B. Tridapalli ${ }^{\mathrm{o}}$, G. Tristram $^{\mathrm{ab}}$, E. Trovato $^{\mathrm{au}}$, M. Tueros ${ }^{\text {bu }}$, R. Ulrich ${ }^{\text {ah }}$, M. Unger ${ }^{\text {ah }}$, M. Urban ${ }^{\text {ac }}$, J.F. Valdés Galicia bd, I. Valiño ${ }^{\text {bu }}$, L. Valore ${ }^{\text {as }}$, A.M. van den Berg ${ }^{\text {bf }}$, E. Varela ba ${ }^{\text {, B. VargasCárdenas }}{ }^{\text {bd }}$, J.R. Vázquez ${ }^{\text {br }}$, R.A. Vázquez ${ }^{\text {bu }}$, D. Veberič ${ }^{\text {bp,bo, }}$,

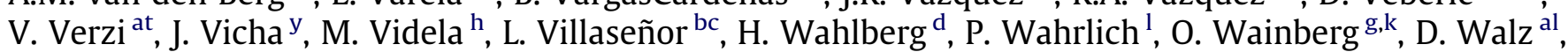
A.A. Watson ${ }^{\text {bw }}$, M. Weber ${ }^{\text {ai }}$, K. Weidenhaupt ${ }^{\text {al }}$, A. Weindl ${ }^{\text {ah }}$, F. Werner ${ }^{\text {ah }}$, S. Westerhoff ${ }^{\text {co }}$, B.J. Whelan ${ }^{\text {i }}$, A. Widom ${ }^{c h}$, G. Wieczorek ${ }^{\text {bj }}$, L. Wiencke ${ }^{\text {bz }}$, B. Wilczyńska ${ }^{\text {bi }}$, H. Wilczyński ${ }^{\text {bi }}$, M. Will ${ }^{\text {ah }}$, C. Williams $^{\text {cl }}$, T. Winchen ${ }^{\text {al }}$, M. Wommer ${ }^{\text {ah }}$, B. Wundheiler ${ }^{\mathrm{g}}$, T. Yamamoto $^{\mathrm{cl}, 4}$, T. Yapici $^{\mathrm{cf}}$, P. Younk ${ }^{\text {an,cd }}$, G. Yuan $^{\mathrm{ce}}$, A. Yushkov ${ }^{\text {bu }}$, B. Zamorano ${ }^{\text {bt }}$, E. Zas ${ }^{\text {bu }}$, D. Zavrtanik ${ }^{\text {bp,bo }}$, M. Zavrtanik ${ }^{\text {bo,bp }}$ I. Zaw $^{\text {cg,5 }}$, A. Zepeda ${ }^{\text {bb }}$, Y. Zhu ${ }^{\text {ai }}$, M. Zimbres Silva ${ }^{\text {ag,p }}$, M. Ziolkowski ${ }^{\text {an }}$

\footnotetext{
${ }^{a}$ Centro Atómico Bariloche and Instituto Balseiro (CNEA-UNCUyo-CONICET), San Carlos de Bariloche, Argentina

${ }^{\mathrm{b}}$ Centro de Investigaciones en Láseres y Aplicaciones, CITEDEF and CONICET, Argentina

${ }^{\mathrm{c}}$ Departamento de Física, FCEyN, Universidad de Buenos Aires y CONICET, Argentina

${ }^{\mathrm{d}}$ IFLP, Universidad Nacional de La Plata and CONICET, La Plata, Argentina

e Instituto de Astronomía y Física del Espacio (CONICET-UBA), Buenos Aires, Argentina

${ }^{\mathrm{f}}$ Instituto de Física de Rosario (IFIR) - CONICET/U.N.R. and Facultad de Ciencias Bioquímicas y Farmacéuticas U.N.R., Rosario, Argentina

${ }^{\mathrm{g}}$ Instituto de Tecnologías en Detección y Astropartículas (CNEA, CONICET, UNSAM), Buenos Aires,Argentina

${ }^{\mathrm{h}}$ National Technological University, Faculty Mendoza (CONICET/CNEA), Mendoza, Argentina

i Observatorio Pierre Auger, Malargüe

j Observatorio Pierre Auger and Comisión Nacional de Energía Atómica, Malargüe, Argentina

${ }^{\mathrm{k}}$ Universidad Tecnológica Nacional - Facultad Regional Buenos Aires, Buenos Aires, Argentina

${ }^{1}$ University of Adelaide, Adelaide, S.A., Australia

${ }^{\mathrm{m}}$ Centro Brasileiro de Pesquisas Fisicas, Rio de Janeiro, RJ, Brazil

${ }^{\mathrm{n}}$ Universidade de São Paulo, Instituto de Física, São Carlos, SP, Brazil

${ }^{\circ}$ Universidade de São Paulo, Instituto de Física, São Paulo, SP, Brazil

${ }^{\mathrm{p}}$ Universidade Estadual de Campinas, IFGW, Campinas, SP, Brazil

${ }^{\mathrm{q}}$ Universidade Estadual de Feira de Santana, Brazil

${ }^{\mathrm{r}}$ Universidade Estadual do Sudoeste da Bahia, Vitoria da Conquista, BA, Brazil

${ }^{s}$ Universidade Federal da Bahia, Salvador, BA, Brazil

${ }^{\mathrm{t}}$ Universidade Federal do ABC, Santo André, SP, Brazil

${ }^{\mathrm{u}}$ Universidade Federal do Rio de Janeiro, Instituto de Física, Rio de Janeiro, RJ, Brazil

${ }^{\mathrm{v}}$ Universidade Federal Fluminense, EEIMVR, Volta Redonda, RJ, Brazil

${ }^{\mathrm{w}}$ Rudjer Boškovi'c Institute, 10000 Zagreb

${ }^{\mathrm{x}}$ Charles University, Faculty of Mathematics and Physics, Institute of Particle and Nuclear Physics, Prague, Czech Republic

${ }^{\mathrm{y}}$ Institute of Physics of the Academy of Sciences of the Czech Republic, Prague, Czech

${ }^{\mathrm{z}}$ Palacky University, RCPTM, Olomouc, Czech Republic

aa Institut de Physique Nucléaire d'Orsay (IPNO), Université Paris 11, CNRS-IN2P3, Orsay

${ }^{\mathrm{ab}}$ Laboratoire AstroParticule et Cosmologie (APC), Université Paris 7, CNRS-IN2P3, Paris, France

${ }^{a c}$ Laboratoire de l'Accélérateur Linéaire (LAL), Université Paris 11, CNRS-IN2P3, Orsay, France

${ }^{a d}$ Laboratoire de Physique Nucléaire et de Hautes Energies (LPNHE), Universités Paris 6 et Paris 7, CNRS-IN2P3, Paris, France
} 
${ }^{\text {ae }}$ Laboratoire de Physique Subatomique et de Cosmologie (LPSC), Université Joseph Fourier, INPG, CNRS-IN2P3, Grenoble, France

af SUBATECH, École des Mines de Nantes, CNRS-IN2P3, Université de Nantes, Nantes, France

${ }^{\text {ag }}$ Bergische Universität Wuppertal, Wuppertal, Germany

${ }^{\text {ah }}$ Karlsruhe Institute of Technology - Campus North - Institut für Kernphysik, Karlsruhe, Germany

${ }^{a i}$ Karlsruhe Institute of Technology - Campus North - Institut für Prozessdatenverarbeitung und Elektronik, Karlsruhe, Germany

aj Karlsruhe Institute of Technology - Campus South - Institut für Experimentelle Kernphysik (IEKP), Karlsruhe, Germany

${ }^{\mathrm{ak}}$ Max-Planck-Institut für Radioastronomie, Bonn, Germany

${ }^{\text {al }}$ RWTH Aachen University, III. Physikalisches Institut A, Aachen, Germany

${ }^{a m}$ Universität Hamburg, Hamburg, Germany

an Universität Siegen, Siegen, Germany

${ }^{\text {ao }}$ Dipartimento di Fisica dell'Università and INFN, Genova, Italy

ap Università dell'Aquila and INFN, L'Aquila

${ }^{\text {aq }}$ Università di Milano and Sezione INFN, Milan, Italy

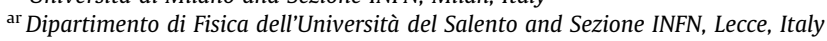

as Università di Napoli "Federico II" and Sezione INFN, Napoli, Italy

at Università di Roma II "Tor Vergata" and Sezione INFN, Roma, Italy

au Università di Catania and Sezione INFN, Catania, Italy

${ }^{\mathrm{av}}$ Università di Torino and Sezione INFN, Torino, Italy

${ }^{\mathrm{aw}}$ Dipartimento di Ingegneria dell'Innovazione dell'Università del Salento and Sezione INFN, Lecce, Italy

ax Istituto di Astrofisica Spaziale e Fisica Cosmica di Palermo (INAF), Palermo, Italy

${ }^{a y}$ Istituto di Fisica dello Spazio Interplanetario (INAF), Università di Torino and Sezione INFN, Torino, Italy

${ }^{\mathrm{az}}$ INFN, Laboratori Nazionali del Gran Sasso, Assergi (L'Aquila), Italy

ba Benemérita Universidad Autónoma de Puebla, Puebla, Mexico

${ }^{\mathrm{bb}}$ Centro de Investigación y de Estudios Avanzados del IPN (CINVESTAV), México, D.F., Mexico

bc Universidad Michoacana de San Nicolas de Hidalgo, Morelia, Michoacan, Mexico

bd Universidad Nacional Autonoma de Mexico, Mexico, D.F., Mexico

be IMAPP, Radboud University Nijmegen

bf Kernfysisch Versneller Instituut, University of Groningen, Groningen, Netherlands

bg Nikhef, Science Park, Amsterdam, Netherlands

${ }^{\text {bh } A S T R O N, \text { Dwingeloo, Netherlands }}$

${ }^{\mathrm{bi}}$ Institute of Nuclear Physics PAN, Krakow

bj University of Łódź, Łódź, Poland

${ }^{\mathrm{bk}}$ LIP and Instituto Superior Técnico, Technical University of Lisbon, Portugal

bl 'Horia Hulubei' National Institute for Physics and Nuclear Engineering, Bucharest-Magurele

${ }^{\mathrm{bm}}$ University of Bucharest, Physics Department, Romania

bn University Politehnica of Bucharest, Romania

bo J. Stefan Institute, Ljubljana, Slovenia

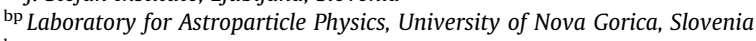

bq Instituto de Física Corpuscular, CSIC-Universitat de València, Valencia, Spain

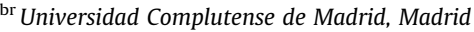

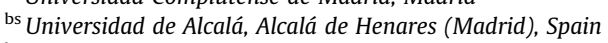

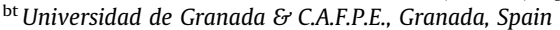

${ }^{\text {bu }}$ Universidad de Santiago de Compostela, Spain

${ }^{\text {bv }}$ Rudolf Peierls Centre for Theoretical Physics, University of Oxford, Oxford, United Kingdom

${ }^{\mathrm{bw}}$ School of Physics and Astronomy, University of Leeds, United Kingdom

${ }^{\mathrm{bx}}$ Argonne National Laboratory, Argonne, IL, USA

by Case Western Reserve University, Cleveland, $\mathrm{OH}, \mathrm{USA}$

${ }^{\text {bz }}$ Colorado School of Mines, Golden, CO, USA

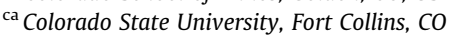

${ }^{\mathrm{cb}}$ Colorado State University, Pueblo, CO, USA

${ }^{\mathrm{cc}}$ Fermilab, Batavia, IL, USA

cd Los Alamos National Laboratory, Los Alamos, NM, USA

${ }^{\mathrm{ce}}$ Louisiana State University, Baton Rouge, LA

${ }^{\mathrm{cf}}$ Michigan Technological University, Houghton, MI, USA

${ }^{\mathrm{cg}}$ New York University, New York, NY, USA

${ }^{\mathrm{ch}}$ Northeastern University, Boston, MA, USA

ci Ohio State University, Columbus, OH, USA

${ }^{\mathrm{cj}}$ Pennsylvania State University, University Park, PA, USA

${ }^{\mathrm{ck}}$ Southern University, Baton Rouge, LA, USA

${ }^{\mathrm{cl}}$ University of Chicago, Enrico Fermi Institute, Chicago, IL, USA

$\mathrm{cm}$ University of Nebraska, Lincoln, NE, USA

${ }^{\mathrm{cn}}$ University of New Mexico, Albuquerque, NM

${ }^{\mathrm{co}}$ University of Wisconsin, Madison, WI, USA

${ }^{\mathrm{cp}}$ University of Wisconsin, Milwaukee, WI, USA

${ }^{\mathrm{cq}}$ Institute for Nuclear Science and Technology (INST), Hanoi, Vietnam

\footnotetext{
* Corresponding author.

E-mail addresses: bianca.keilhauer@kit.edu (B. Keilhauer), martin.will@kit.edu (M. Will), auger_spokepersons@fnal.gov.

1 now at University of Maryland

2 now at Université de Lausanne

3 Deceased

4 at Konan University, Kobe, Japan

5 now at NYU Abu Dhabi
} 


\section{A R T I C L E I N F O}

\section{Article history:}

Received 2 August 2011

Received in revised form 7 December 2011

Accepted 27 December 2011

Available online 11 January 2012

\section{Keywords:}

Cosmic rays

Extensive air showers

Atmospheric monitoring

Atmospheric models

\begin{abstract}
A B S T R A C T
Atmospheric conditions at the site of a cosmic ray observatory must be known for reconstructing observed extensive air showers. The Global Data Assimilation System (GDAS) is a global atmospheric model predicated on meteorological measurements and numerical weather predictions. GDAS provides altitude-dependent profiles of the main state variables of the atmosphere like temperature, pressure, and humidity. The original data and their application to the air shower reconstruction of the Pierre Auger Observatory are described. By comparisons with radiosonde and weather station measurements obtained on-site in Malargüe and averaged monthly models, the utility of the GDAS data is shown.
\end{abstract}

(C) 2012 Elsevier B.V. All rights reserved.

\section{Introduction}

The Pierre Auger Observatory $[1,2]$ is located near the town of Malargüe in the province of Mendoza, Argentina. At the site, at the base of the Andes mountains, two well-established measurement techniques are combined to measure extensive air showers with energies above some $10^{17} \mathrm{eV}$. The hybrid detector consists of a surface detector (SD) array and five fluorescence detector (FD) buildings. Each of the slightly more than 1600 SD stations is a water-filled Cherenkov detector, measuring the secondary particles of air showers that reach the ground. The detectors of the array are spaced by $1.5 \mathrm{~km}(750 \mathrm{~m}$ in a small infill area in the western part of the array) and provide the lateral particle distribution around a shower core. Four FD buildings comprise six telescopes each and one FD enhancement installation consists of three telescopes. In each FD telescope, the UV light emitted by excited nitrogen molecules along the shower track is collected by a large segmented mirror and reflected onto a camera composed of 440 PMTs. With this measurement, the geometry and the longitudinal profile of the shower can be obtained.

For the reconstruction of extensive air showers, the optical properties of the atmosphere at the site of the observatory have to be known. This is particularly true for reconstructions based on data obtained with the fluorescence technique [3], but also impacts upon data collected with the surface detectors [4]. The detection of clouds is an important task of the atmospheric monitoring systems. Clouds can obstruct or - through scattering of the intense Cherenkov light - amplify the apparent fluorescence light before it reaches the FD. To eliminate data recorded in cloudy conditions from physics analyses, lidar stations and infrared cloud cameras are installed at each FD station of the Pierre Auger Observatory. These instruments scan the fields of view of the fluorescence detectors several times per hour during data taking periods to measure the cloud coverage and the base height of clouds [5]. The vertical profile of the aerosol optical depth is measured once every hour using vertical laser shots from two facilities near the center of the array. Using the calibrated laser energy and the amount of light scattered out of the beam towards the FDs, the amount of aerosols can be estimated [3]. Weather conditions near ground, and the height-dependent atmospheric profiles of temperature, pressure and water vapor pressure are relevant for several Auger Observatory measurements. E.g., these parameters affect the production of fluorescence light by excited nitrogen molecules at the shower track, and the Rayleigh scattering of the light between the air shower and detector. Atmospheric conditions are measured by intermittent meteorological balloon radio soundings. Additionally, ground-based weather stations measure surface data continuously. The profiles from the weather balloons were averaged to obtain local models, called (new) Malargüe Monthly Models [3]. Since March 2009, the atmospheric monitoring system has been upgraded with the implementation of a rapid monitoring system [6]. Part of the new program was the measurement of atmospheric profiles with radio soundings shortly after the detection of particularly high-energy air showers, a system called Balloon-the-Shower (BtS). This enables a high-quality reconstruction of the most interesting events.

However, performing radio soundings and applying these data to air shower analyses is not straightforward. Very critical aspects are the time of the weather balloon ascent and the data validity period. Furthermore, performing radio soundings, in particular within BtS, imposes a large burden on the collaboration. Therefore, we investigate the possibility of using data from the Global Data Assimilation System (GDAS), a global atmospheric model, for the site of the Auger Observatory. The data are publicly available free of charge via READY (Real-time Environmental Applications and Display sYstem). Each data set contains all the main state variables with their dependence on altitude with a validity period of 180 min for each data set.

Key aspects of the impact of the profiles of atmospheric state variables on the development and detection of extensive air showers are discussed briefly (Section 2). We motivate the necessity of more reliable atmospheric profiles by a discussion about the data validity period of weather balloons (Section 3), describe the content and processing of the GDAS data (Section 4) and compare them to local measurements (Section 5). The new atmospheric data are implemented in the data processing and simulation framework of the Auger Observatory for an analysis of reconstructed air showers (Section 6).

\section{Impact of atmospheric state variables on the development and detection of extensive air showers}

Varying atmospheric conditions in terms of state variables like temperature, pressure and humidity, may alter the development and, in particular, the detection of extensive air showers. Here, different aspects relevant to the analysis of air showers at the Pierre Auger Observatory are discussed.

The air fluorescence emission excited by the passage of an air shower depends on pressure, temperature, and humidity [7]. The collisional de-excitation of excited nitrogen molecules by other molecules of the atmosphere like nitrogen, oxygen, and water vapor counteracts the de-excitation of the molecules via radiation. These quenching processes are pressure and temperature dependent as described by kinetic gas theory, and dependent on the water vapor content in air. Furthermore, the collisional cross sections for nitrogen-nitrogen and nitrogen-oxygen collisions follows a power law in temperature, $\sigma \propto T^{\alpha}$. Most recent experimental data indicate a negative exponent $\alpha$. In reconstructions of air shower data from the Auger Observatory, the fluorescence yield with its dependence on atmospheric conditions is described using experimental results from the AIRFLY experiment $[8,9]$. The absolute calibration of the main fluorescence emission at $337.1 \mathrm{~nm}$ is taken from Nagano et al. [10]. The dependence of the fluorescence yield 
on atmospheric conditions translates to an atmospheric dependence of the reconstructed cosmic ray energy and the depth of shower maximum, the latter being an indicator for the mass of the primary cosmic ray particle. Even short-term variations of the atmosphere may introduce noticeable effects on these reconstructed parameters.

Besides the fluorescence emission, the pressure, temperature and humidity profiles of the atmosphere are important for other aspects of the reconstruction of data collected by the FD. These include the conversion between geometrical altitudes and atmospheric depth; the treatment of Cherenkov emission from air showers; and the transmission of the produced photons from the air shower to the FD:

- The air shower development is governed by the interactions and decays of the secondary particles. These processes are largely determined by the atmospheric depth $X$, the total column density of atmospheric matter traversed by the air shower at a given point. $X$ is calculated by integrating the density of air from the top of the atmosphere, along the trajectory of the shower through the gas. The observation of the longitudinal shower profiles by fluorescence telescopes is based on geometrical altitudes $h$. Thus, geometrical altitudes must be converted into atmospheric depth by taking into account the actual air density profile $\rho(h)$ at the site of the Observatory, and the zenith angle $\theta$ of the trajectory of the shower,

$$
X\left(h_{0}\right)=\frac{1}{\cos \theta} \int_{h_{0}}^{\infty} \rho(h) \mathrm{d} h .
$$

- The secondary particles in extensive air showers travel faster than the speed of light in air. As a result, they induce the emission of Cherenkov light in a narrow, forward-beamed cone. Some of this light in the UV range may be - depending on the shower geometry relative to the FD telescope - detected together with the fluorescence light. To effectively subtract the Cherenkov photons from the total number of photons detected, the amount of Cherenkov light emitted by the air shower must be estimated. The Cherenkov yield depends on the refractive index $n$ of the air, which itself depends on the wavelength of the emitted light as well as the temperature, pressure and humidity $[11,12]$. Parameterized formulae for the refractive index of dry air, $\mathrm{CO}_{2}$ and water vapor are used to calculate a total refractive index,

$$
\begin{aligned}
n_{\mathrm{tot}}-1= & \left(n_{\mathrm{dry}}-1\right) \cdot \frac{\rho_{\mathrm{dry}}}{\rho_{\mathrm{air}}}+\left(n_{\mathrm{CO}_{2}}-1\right) \cdot \frac{\rho_{\mathrm{CO}_{2}}}{\rho_{\mathrm{air}}} \\
& +\left(n_{\mathrm{w}}-1\right) \cdot \frac{\rho_{\mathrm{w}}}{\rho_{\mathrm{air}}} .
\end{aligned}
$$

The refractive index of each component is weighted with its density, which can be calculated using the number density and the molar mass of the constituent. Finally, the effect of the decreasing number density with altitude is parameterized [13] as a function of pressure $p$ and temperature $\vartheta$ in ${ }^{\circ} \mathrm{C}$,

$$
n_{\mathrm{air}}-1=\left(n_{\mathrm{tot}}-1\right) \cdot p \cdot \frac{1+p \cdot(61.3-\vartheta) \cdot 10^{-10}}{96095.4 \cdot(1+0.003661 \cdot \vartheta)}
$$

- Between the production of fluorescence and Cherenkov light in the air shower and the detection at the FD telescope, the light is scattered by molecules in the atmosphere. The transmission of light depends on the Rayleigh cross section [14],

$$
\sigma_{\mathrm{R}}(\lambda, p, T, e)=\frac{24 \pi^{3}}{\lambda^{4} \cdot N^{2}} \cdot\left(\frac{n_{\mathrm{air}}^{2}-1}{n_{\mathrm{air}}^{2}+2}\right)^{2} \cdot F_{\mathrm{air}}(\lambda, p, e),
$$

where $\lambda$ is the wavelength in $\mathrm{m}$ and $N$ the atmospheric molecular density, measured in molecules per $\mathrm{m}^{-3} . F_{\text {air }}$ is the King factor that accounts for the anisotropy in the scattering introduced by nonspherical scatter centers, which depends slightly on pressure and humidity. The refractive index $n_{\text {air }}$ depends on several atmospheric state variables, see Eq. (3).

The last three itemized effects on the reconstruction of extensive air showers can be taken sufficiently into account by using a proper description of the atmospheric state, e.g., the local atmospheric monthly models derived from multi-year meteorological balloon radio soundings (see Section 3). They affect the reconstruction results of air shower data, mainly primary energy and position of shower maximum, only by marginally broadening the uncertainties without any significant systematic shifts.

However, in the case of the earlier discussed fluorescence emission process and its atmospheric variability, systematic alterations of the reconstruction results may be seen together with increased uncertainties, even for short-term variations of the atmospheric parameters. Finally, after this discussion on atmospheric influences on FD analysis, it should be noted that uncertainties in the surface detector signals introduced by varying atmospheric conditions close to the ground are well understood and quantified [4].

\section{Validity of radio soundings}

Since August 2002, meteorological radio soundings have been performed above the Pierre Auger Observatory to measure altitude-dependent profiles of atmospheric variables, mainly pressure, temperature, and relative humidity. Regular measurements were done until December 2008 in order to collect data for all months. After applying selection criteria, 261 profiles from the middle of 2002 until the end of 2008 could be used to build the new Malargüe Monthly Models [3]. Starting in March 2009, the radio soundings became part of the rapid atmospheric monitoring system known as the Balloon-the-Shower $(B t S)$ program $[6,15]$. A fast online air shower reconstruction with subsequent quality selections is used to trigger the launch of a weather balloon by a local technician.

A procedure was developed to find the period of time for which the data measured during the ascent of a weather balloon give a good description of the atmospheric conditions at the Pierre Auger Observatory. The 3-dimensional atmospheric conditions before and after a weather balloon ascent are unknown but data from local weather stations may help to identify stable periods or trends towards rapidly changing conditions. Every active weather station is used as an independent source of data, no matter how many stations contribute information during the period of the weather balloon ascent. For each station, the maximum variations of the temperature, the pressure, and the humidity are obtained for the

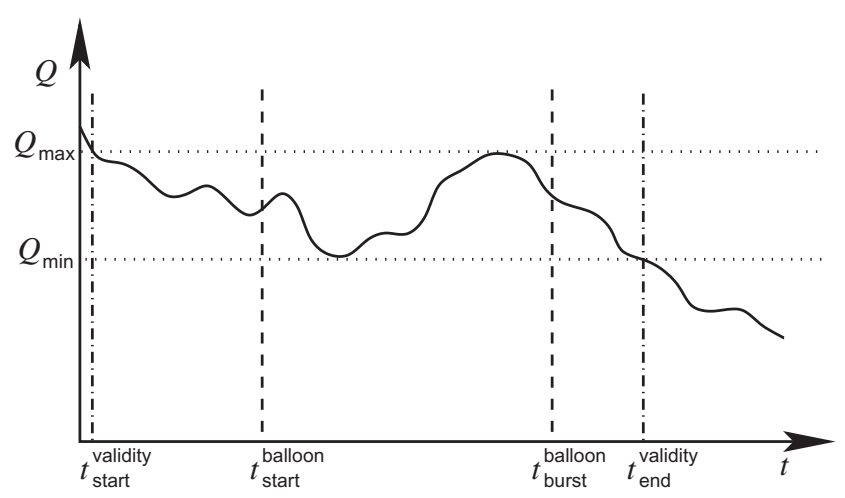

Fig. 1. Schematic drawing of the procedure to find an extended time period of validity for data from weather balloons based on data measured by ground-based weather stations for typical atmospheric conditions. For details see text. 

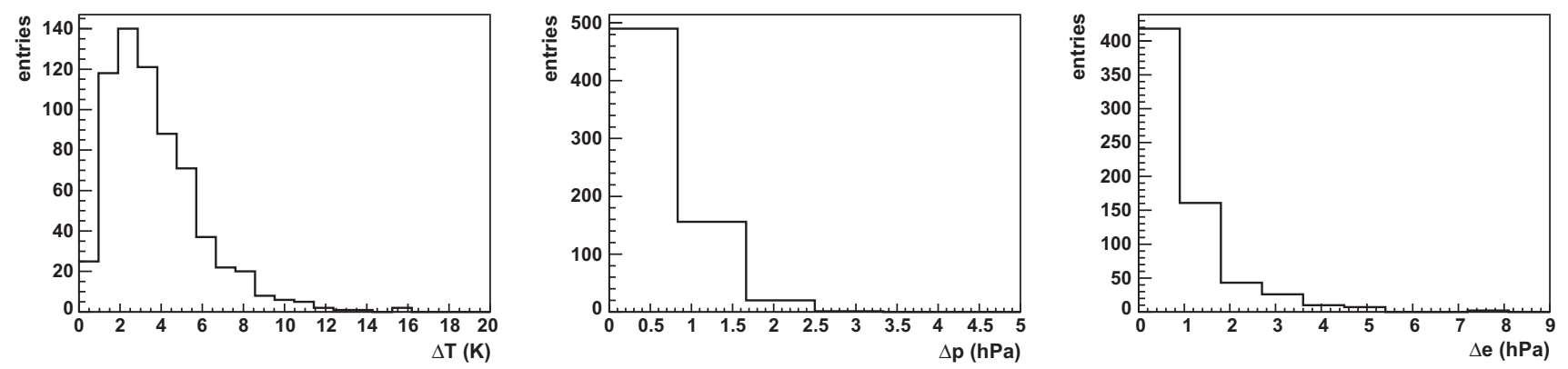

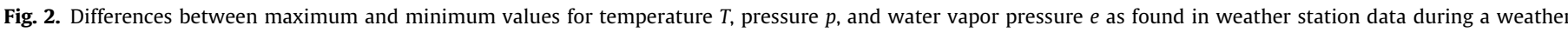

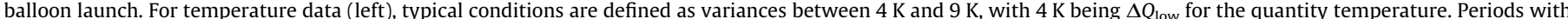

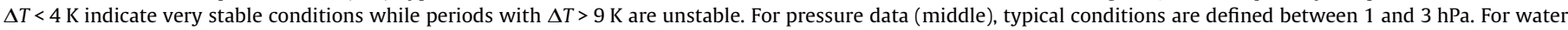

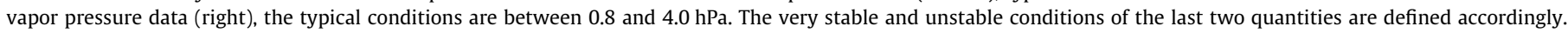

duration of the corresponding weather balloon launch defined as the time between the start of the weather balloon and the burst of the balloon, see Fig. 1.

The difference $\Delta Q$ - with $Q$ being temperature $T$, pressure $p$, or water vapor pressure $e$ - between maximum $\left(Q_{\max }\right)$ and minimum $\left(Q_{\text {min }}\right)$ values of every station during weather balloon flights can be seen in Fig. 2. From these histograms, periods with very stable conditions $\left(\Delta Q<\Delta Q_{\text {low }}\right)$, with typical conditions, and with unstable conditions are defined for each quantity $Q$ (see caption of Fig. 2).

For typical conditions, the data of the weather stations are scanned before and after the time of the balloon ascent for each quantity $Q$ and the time at which any quantity leaves the range between $Q_{\min }$ and $Q_{\max }$ is determined. This time period gives the validity time period of the radio sounding for every active weather station. For launches performed during very stable conditions, the differences in weather station data are quite small. Thus, only small variations beyond the narrow interval would indicate the end of validity, imposing very strict cuts on this type of launch. For unstable conditions, the large $\Delta Q$ values could result in quite long extended periods of validity. Since both cases result in inappropriate validity periods, two special criteria for each quantity are found in addition to the typical case. For very stable conditions, $Q_{\max / \min }$ are redefined to $\widetilde{Q}_{\max / \min }=\bar{Q} \pm Q_{\text {low }} / 2$, where $\bar{Q}$ is the mean of the interval $Q_{\min }$ to $Q_{\max }$ and $Q_{\text {low }}$ is $4 \mathrm{~K}$ in the case of temperature data, $1 \mathrm{hPa}$ for pressure data, and $0.8 \mathrm{hPa}$ for water vapor pressure data. After definition of $\widetilde{Q}_{\max / \min }$, the same procedure as for the typical conditions is applied. In case of unstable conditions, the validity time period is set to the time period during which the weather balloon ascended.

The average duration of a weather balloon ascent was about $100 \mathrm{~min}$. A validity time period of $200 \mathrm{~min}$ on average is given by the local weather station data as described above. Applying this procedure, about half of the cosmic ray events which triggered the BtS program are observed at times not covered by the period of validity of the corresponding balloon launch.

Until its termination at the end of 2010, many details of local atmospheric conditions could be studied with the BtS program. The obtained atmospheric profiles can be applied to improve the reconstruction of the most interesting, high-energy air showers. However, the data are not suitable for application to the standard reconstruction because of their short period of validity. Only very few air shower events would be covered by atmospheric profiles from radio soundings.

\section{Global Data Assimilation System (GDAS)}

In the field of numerical weather prediction, data assimilation is the adjustment of the development within a model to the real behavior of the atmosphere as found in meteorological observa-

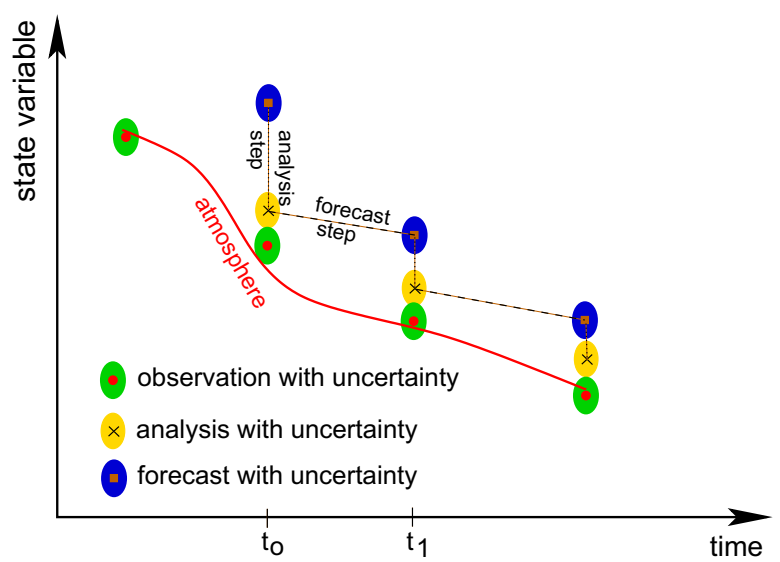

Fig. 3. Schematic of the data assimilation process. Figure adopted in a modified form from [17].

tions [16]. The atmospheric models describe the atmospheric state at a given time and position. Three steps are needed to perform a full data assimilation:

1. Collect data from meteorological measuring instruments placed all over the world. These instruments include weather stations on land, ships, and airplanes as well as radiosondes and weather satellites.

2. Use a short-term forecast from a previous iteration of the numerical weather prediction together with the measurements to describe the current situation. This additional information is needed because the available observations alone are not sufficient. The forecast or first guess adds more information to the system, namely all knowledge of atmospheric behavior expressed in mathematical model equations. The models use non-linear differential equations based on thermodynamics and fluid dynamics.

3. Adjust the model output to the measured atmospheric state. The resulting 3-dimensional image of the atmosphere is called analysis.

A schematic showing the principle of data assimilation is given in Fig. 3. At a given time $t_{0}$, the observations provide the value of a state variable. A model forecast for this variable from a previous iteration exists for the same time. The analysis step combines observation and forecast to describe the current state better than the forecast. This analysis is the initial point for the weather prediction model to create the forecast for a later time $t_{1}$. 
Table 1

Vertical levels of pressure surfaces. The actual height for each pressure level is given in the data file. For reference, the USStdA height is also listed in this table. Pressure level 0 contains the surface values.

\begin{tabular}{llllll}
\hline Level & Pressure $[\mathrm{hPa}]$ & Height $[\mathrm{km}]$ & Level & Pressure $[\mathrm{hPa}]$ & Height $[\mathrm{km}]$ \\
\hline 23 & 20 & 26.4 & 11 & 600 & 4.2 \\
22 & 50 & 20.6 & 10 & 650 & 3.6 \\
21 & 100 & 16.2 & 9 & 700 & 3.0 \\
20 & 150 & 13.6 & 8 & 750 & 2.5 \\
19 & 200 & 11.8 & 7 & 800 & 1.9 \\
18 & 250 & 10.4 & 6 & 850 & 1.5 \\
17 & 300 & 9.2 & 5 & 900 & 1.0 \\
16 & 350 & 8.2 & 4 & 925 & 0.8 \\
15 & 400 & 7.2 & 3 & 950 & 0.5 \\
14 & 450 & 6.3 & 2 & 975 & 0.3 \\
13 & 500 & 5.6 & 1 & 1000 & 0.1 \\
12 & 550 & 4.9 & 0 & Surface & \\
\hline
\end{tabular}

\subsection{GDAS data}

The Global Data Assimilation System [18] is an atmospheric model developed at NOAA's ${ }^{6}$ National Centers for Environmental Prediction (NCEP). It provides an analysis four times a day $(0,6$, 12 , and 18 UTC) and a 3-, 6- and 9-hour forecast. The numerical weather prediction model used in the GDAS is the Global Forecast System (GFS).

3-hourly data are available at 23 constant pressure levels - from $1000 \mathrm{hPa}$ (roughly sea level) to $20 \mathrm{hPa}(\approx 26 \mathrm{~km})$ - on a global $1^{\circ}$ spaced latitude-longitude grid $\left(180^{\circ}\right.$ by $\left.360^{\circ}\right)$. Each data set is complemented by data for the surface level. The data are stored in weekly files and made available online [18]. In Table 1, the level indices corresponding to each data level are listed. For reference, the altitude from the US Standard Atmosphere 1976 (USStdA) [19] is also given in the table. The actual height of the pressure level is stored in the data file. GDAS data are available starting January 2005 . There are two periods without data in the sets. The first two weeks of May 2005 and weeks 3 and 4 of November 2005 are missing. Other than that, the record is complete up to the present time (end of November 2011).

Because of the lateral homogeneity of the atmospheric variables across the Auger array [3], only one location point is needed to describe the atmospheric conditions. In Fig. 4, the available GDAS grid points are marked as red crosses on a map together with a map of the surface and fluorescence detectors of the Auger Observatory. The grid point at $35^{\circ}$ south and $69^{\circ}$ west was chosen, at the north-eastern edge of the surface detector array. The two points to the west of the array are in the foothills of the Andes mountains and therefore not suitable. The point to the south-east of the array is quite far away and with a surface height of $1685 \mathrm{~m}$ a.s.l., it is also too high. Nevertheless, the profiles at this point are very similar to those at the chosen point, on average differing by less than $1{ }^{\circ} \mathrm{C}$ in temperature and less than $0.3 \mathrm{hPa}$ in water vapor pressure at all altitudes, confirming the homogeneity.

The height at which the surface data are given changes over the years for the selected grid point. Starting in January 2005, the surface altitude is $1831.29 \mathrm{~m}$ above sea level. On May 31, 2005, the surface height changes to $1403.38 \mathrm{~m}$, and on August 22, 2006 it goes down further to $1328.68 \mathrm{~m}$ and stays within a few centimeters of this value until July 27, 2010, when it changes to $1404.65 \mathrm{~m}$. In Fig. 5, the surface height provided by the GDAS data sets is shown between January 2005 and December 2010. For reference, the altitudes of the lowest SD tank $(1331.05 \mathrm{~m})$ and the highest and lowest FD buildings (1712.3 $\mathrm{m}$ and $1416.2 \mathrm{~m}$ ) are also shown. The reasons for these changes are regular improvements of

\footnotetext{
${ }^{6}$ National Oceanic and Atmospheric Administration.
}

the models and calculations used to produce the GDAS profiles, or resolution changes in the meteorological model [20]. These changes can occur again in the future, so the surface height of the data has to be monitored for undesired changes [21].

For air shower analysis, only data above ground level in Malargüe are interesting. Therefore, we only use data from the surface and from pressure levels 6 and above. The data from beginning of January to the end of May 2005 have a surface height of around $1800 \mathrm{~m}$. This is even above the height of the highest FD building at Coihueco. We decided not to attempt an extrapolation down to the actual ground level of around $1300 \mathrm{~m}$ and discard these data. Therefore, the first data set we use is from June 1, 2005 at 0:00 UTC.

\subsection{Preprocessing of data}

For air shower analyses, several types of information are stored in databases such as the one describing the state variables of the atmosphere. It contains values for temperature, pressure, relative humidity, air density, and atmospheric depth at several altitude levels. The first three quantities and the altitude are directly available in the GDAS data. Air density and atmospheric depth must be calculated. The surface data contain ground height, pressure at the ground, and relative humidity $2 \mathrm{~m}$ above ground. Two temperature values are given, one at the surface and one $2 \mathrm{~m}$ above ground. We decided to use the latter since we use it together with the relative humidity, which is also given $2 \mathrm{~m}$ above ground, to calculate water vapor pressure.

In the GDAS data, the altitude is given in geopotential meters with respect to a geoid (mean sea level). In the air shower analysis framework of the Auger Observatory, geometric heights with respect to the WGS-84 ellipsoid are used. To move from geoid to ellipsoid, a constant value of $26 \mathrm{~m}$ that arises from the geographic location of the Auger Observatory must be added to the height values of the model. The second step is to convert from geopotential height $h$ to geometric altitude $z$ (both measured in $\mathrm{m}$ ),

$$
\begin{aligned}
z(h, \Phi)= & (1+0.002644 \cdot \cos (2 \Phi)) \cdot h+(1+0.0089 \\
& \cdot \cos (2 \Phi)) \cdot \frac{h^{2}}{6245000},
\end{aligned}
$$

where $\Phi$ is the geographical latitude [22].

To calculate the air density, the relative humidity must be converted into water vapor pressure, the partial pressure of water in air in Pa. This conversion depends on air temperature. The following approximation of the empirical Magnus formula is used in these calculations:

$$
\begin{aligned}
& e=\frac{u}{100 \%} \cdot 6.1070 \cdot \exp \left(\frac{17.15 \cdot \vartheta}{234.9+\vartheta}\right), \quad \vartheta \geqslant 0{ }^{\circ} \mathrm{C}, \\
& e=\frac{u}{100 \%} \cdot 6.1064 \cdot \exp \left(\frac{21.88 \cdot \vartheta}{265.5+\vartheta}\right), \quad \vartheta<0{ }^{\circ} \mathrm{C},
\end{aligned}
$$

where $u$ is the relative humidity in $\%$ and $\vartheta$ is the temperature in ${ }^{\circ} \mathrm{C}$. Now, the air density in $\mathrm{kg} \mathrm{m}^{-3}$ can be calculated with

$\rho=\frac{p \cdot M_{\mathrm{air}}}{R \cdot T}$,

where $p$ is the pressure in Pa, $T$ is the temperature in $\mathrm{K}, M_{\mathrm{air}}$ is the molar mass of air in $\mathrm{kg} \mathrm{mol}^{-1}$ and $R$ is the universal gas constant $8.31451 \mathrm{~J} \mathrm{~K}^{-1} \mathrm{~mol}^{-1}$. Moist air can be separated into three components to calculate its molar mass: dry air, water vapor and carbon dioxide. The molar mass of moist air is the sum of the molar masses of the components, weighted with the volume percentage of that component,

$M_{\mathrm{air}}=M_{\mathrm{dry}} \cdot \varphi_{\mathrm{dry}}+M_{\mathrm{w}} \cdot \varphi_{\mathrm{w}}+M_{\mathrm{CO}_{2}} \cdot \varphi_{\mathrm{CO}_{2}}$. 


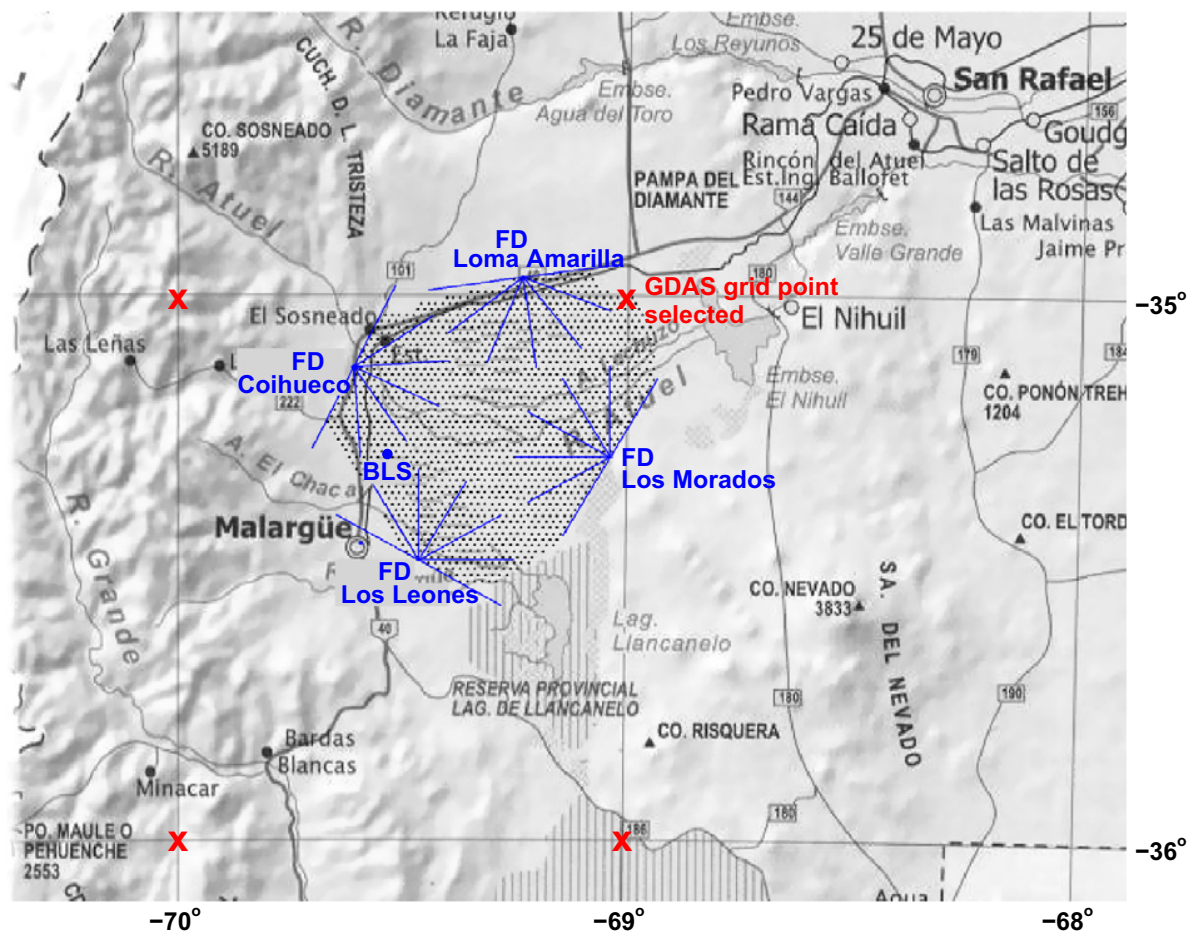

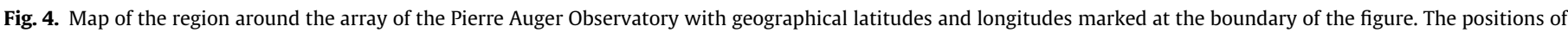

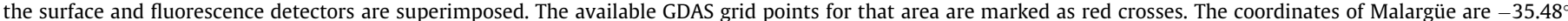
(south) and $-69.58^{\circ}$ (west), the selected GDAS grid point is $-35^{\circ}$ (south) and $-69^{\circ}$ (west).

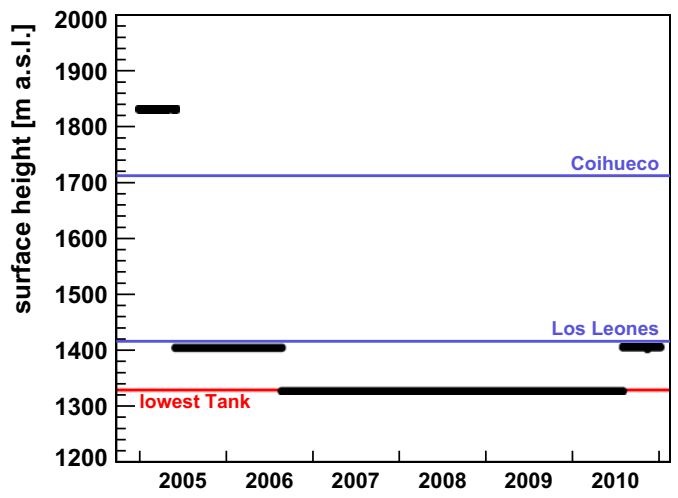

Fig. 5. The surface height of the GDAS data between January 2005 and December 2010. For reference, the red line marks the height of the lowest tank in the array, and the blue lines represent the height of the FD buildings at Los Leones and Coihueco.

The molar masses for dry air, water vapor and $\mathrm{CO}_{2}$ are 0.02897 , 0.04401 and $0.01802 \mathrm{~kg} \mathrm{~mol}^{-1}$, respectively. The volume percentage of $\mathrm{CO}_{2}$ is taken as $385 \mathrm{ppmv}$, the percentage of water $\varphi_{\mathrm{w}}$ is the partial pressure $e$ of water vapor divided by the pressure $p$, and dry air makes up the rest, $\varphi_{\mathrm{dry}}=1-\varphi_{\mathrm{w}}-\varphi_{\mathrm{CO}_{2}}$. The atmospheric depth can be calculated by integrating the air density in $\mathrm{kg} \mathrm{m}^{-3}$ downward along a vertical track in the atmosphere, starting at infinity

$X\left(h_{0}\right)=\int_{h_{0}}^{\infty} \rho \mathrm{d} h$.

Here, the atmospheric depth is calculated in $\mathrm{kg} \mathrm{m}^{-2}$. In the astroparticle community, the unit $\mathrm{g} \mathrm{cm}^{-2}$ is more common and will be used in this paper.

Since the GDAS data only go up to around $25-30 \mathrm{~km}$, we have to approximate the atmospheric depth at the top of the data profile using $X=p / g$ and integrate numerically from that height down to ground level. $g$ is the gravitational acceleration with dependence on altitude and geographical latitude [23],

$$
\begin{aligned}
g(h, \Phi)= & g_{0}(\Phi)-\left(3.085462 \cdot 10^{-4}+2.27 \cdot 10^{-7} \cdot \cos (2 \Phi)\right) \\
& \cdot h+\left(7.254 \cdot 10^{-11}+1.0 \cdot 10^{-13} \cdot \cos (2 \Phi)\right) \cdot h^{2} \\
& -\left(1.517 \cdot 10^{-17}+6 \cdot 10^{-20} \cdot \cos (2 \Phi)\right) \cdot h^{3},
\end{aligned}
$$

where $g$ is in $\mathrm{cm} \mathrm{s}^{-2}$ and $g_{0}(\Phi)$ is the acceleration at sea level with dependence on latitude,

$$
\begin{aligned}
g_{0}(\Phi)= & 980.6160 \cdot(1-0.0026373 \cdot \cos (2 \Phi)+0.0000059 \\
& \left.\cdot \cos ^{2}(2 \Phi)\right) .
\end{aligned}
$$

The integration is done by interpolating the density every $200 \mathrm{~m}$ and using the trapezoidal rule to approximate the integral.

For the simulation and reconstruction of air showers, the description of the atmospheric parameters should ideally range from ground level to the top of the atmosphere. GDAS provides data between about 1400 and 30,000 $\mathrm{m}$. All profiles are extended up to $100,000 \mathrm{~m}$ - the approximate boundary to outer space using the US Standard Atmosphere 1976 [19] which describes the conditions above $30,000 \mathrm{~m}$ reasonably well. Below $1400 \mathrm{~m}$, pressure, atmospheric depth, density, and water vapor pressure are exponentially extrapolated down to $1000 \mathrm{~m}$ based on the lowest two data points. The temperature profile is extrapolated linearly. Both extensions are outside the field of view of all FD stations.

\section{GDAS vs. local measurements}

To validate the quality of the GDAS data and to verify its applicability for air shower reconstructions at the Auger Observatory, GDAS data are compared with local measurements - atmospheric soundings with weather balloons and ground-based weather stations. The new Malargüe Monthly Models (nMMM) are also shown 
in some comparisons as a reference since they were the standard profiles used in reconstructions until recently.

\subsection{GDAS vs. soundings with weather balloons}

Local atmospheric soundings have been performed above the array of the observatory since 2002, but not on a regular basis. In the beginning, several week-long campaigns of launches were performed. Then, a pre-determined schedule was used to coordinate launches during and between dark periods with FD data taking and finally, with the BtS program, soundings are triggered by particularly high-energy events during FD data taking only. Most of the balloons were launched from the Balloon Launching Station (BLS, see Fig. 4). To provide a set of atmospheric data for every measured air shower, the soundings were averaged to form monthly mean profiles. The current version of these Malargüe Monthly Models (nMMM) were compiled in early 2009. The models on average describe the atmosphere reasonably well, but show considerable fluctuations when compared to the actual sounding data [3]. The uncertainties of the profile for each variable are given by the standard error of the variation within each month together with the absolute uncertainties of the sensors measuring the corresponding quantity. For atmospheric depth profiles, a piecewise fitting procedure is performed to ensure a reliable application of these parameterizations to air shower simulation programs. An additional uncertainty is included which covers the quality of the fitting procedure.

In Fig. 6, the GDAS data are compared with the measured radiosonde data between 2005 and 2008. The comparison between the radiosonde data and the monthly mean profiles is also shown. The monthly mean profiles are averaged from the sounding data until end of 2008, thus, they fit local sounding data of this period very well, see red squares in Fig. 6 . The error bars denote the RMS of the differences at each height. The wave-like shape in the difference graph for the atmospheric depth $X$ is driven by the piecewise parameterization of $X$ in the nMMM. Above around $5 \mathrm{~km}$, the GDAS data deviate only marginally from the measured data (black dots in Fig. 6). Closer to the ground, slightly larger differences become apparent. The measured temperature is consistently higher than the model temperature. However, this might be caused by two problems acting in the same direction. For our local radio soundings, the temperature sensor might be quite often not properly acclimatized to outside conditions but is affected by the inside temperature of the Balloon Launching Station. It takes some minutes to overcome this effect during which time the weather balloon is already launched. When compiling the nMMM, only temperature data at $1600 \mathrm{~m}$ a.s.l. and above were taken from the radio soundings. To extrapolate to lower altitudes, a fit to these sounding data combined with data from local weather stations at $1401 \mathrm{~m}, 1420 \mathrm{~m}$, $1423 \mathrm{~m}, 1483 \mathrm{~m}$, and $1719 \mathrm{~m}$ was performed. However, data from the weather stations might also be influenced by the direct surface conditions beneath, since they are not standardized meteorological stations. It is also possible that the GDAS model does not adequately describe the heated surface of the elevated plain of the Pampa Amarilla and tends to assume free-atmosphere conditions. The pressure data of both models are in good agreement. The water vapor pressure fits almost perfectly, although the model values close to ground are both too low compared to real data which can be traced back to the difficult handling of humidity in general. In particular, the pressure differences propagate into the atmospheric depth where we see deviations from the measured data on the same scale as for the monthly mean profiles.

In the comparison displayed in Fig. 7, only radiosonde data from 2009 and 2010 are used in order to illustrate the strength of the GDAS model data. The nMMM are completely independent of this set of radio soundings. The most obvious changes compared with Fig. 6 are the worse descriptions of actual radio soundings with monthly mean profiles. Even though the uncertainties become lar-
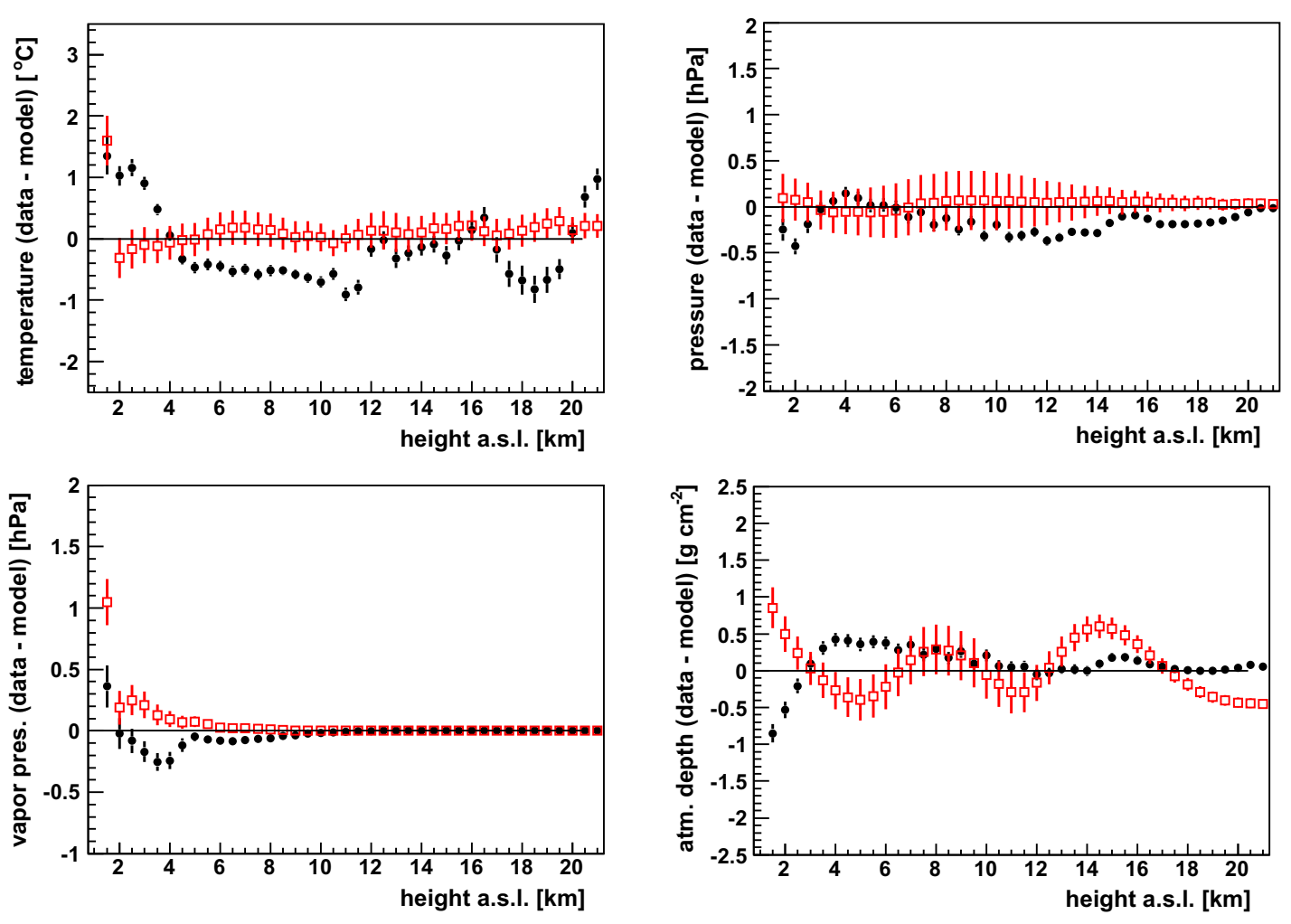

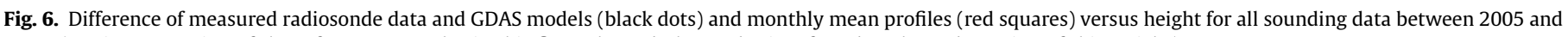
2008. (For interpretation of the references to color in this figure legend, the reader is referred to the web version of this article.) 

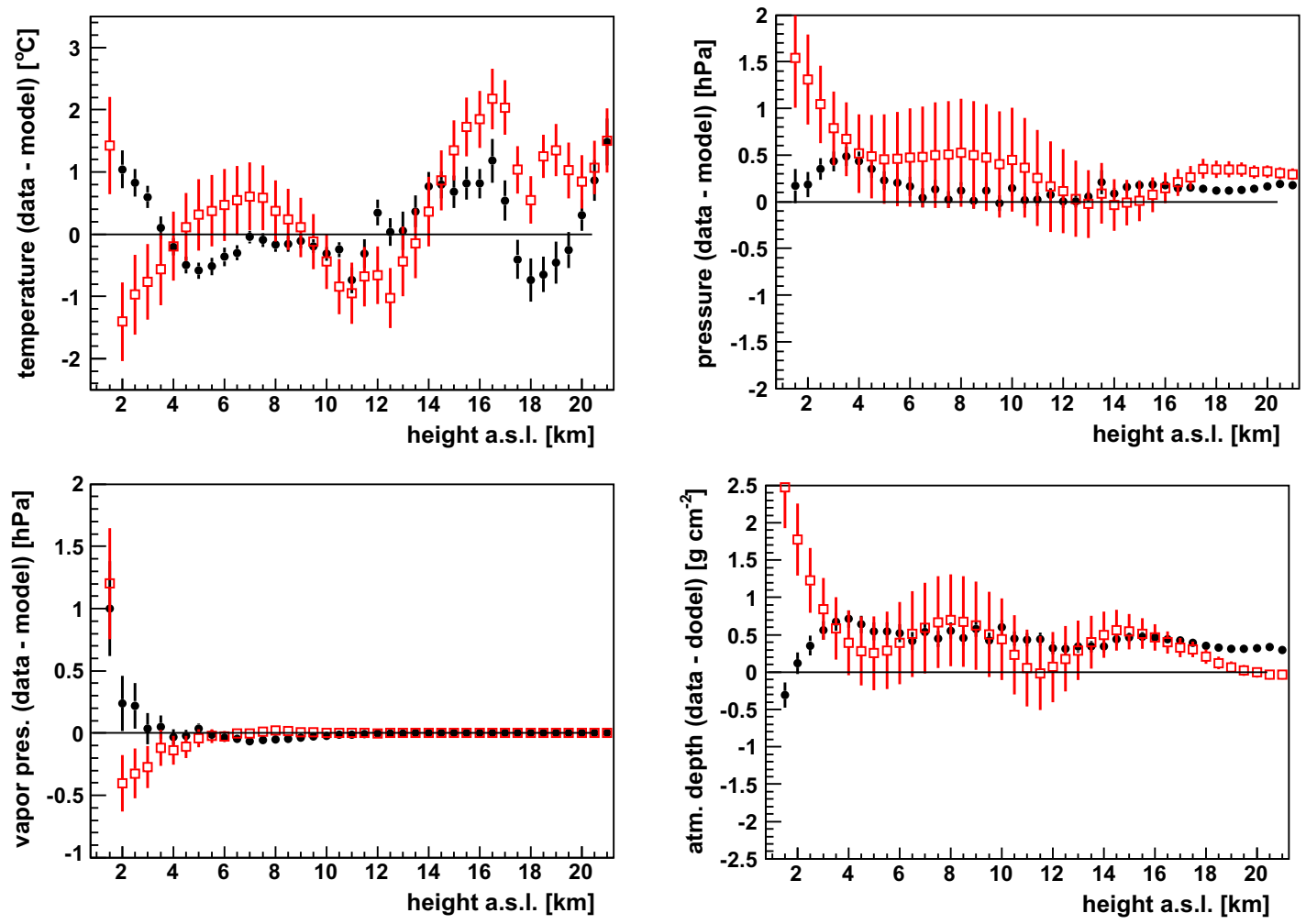

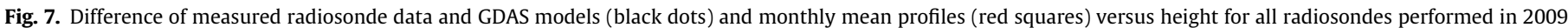
and 2010. (For interpretation of the references to color in this figure legend, the reader is referred to the web version of this article.)
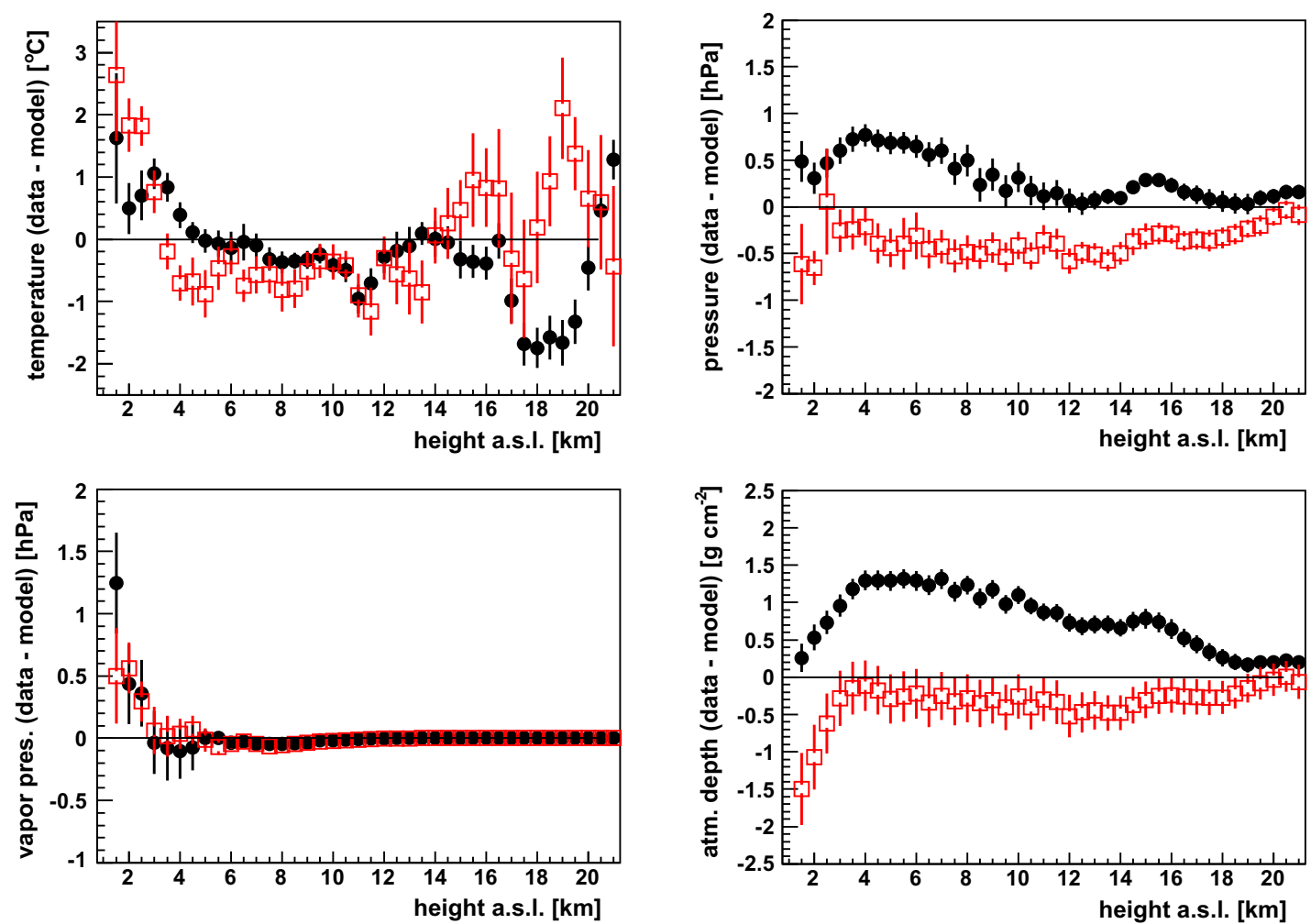

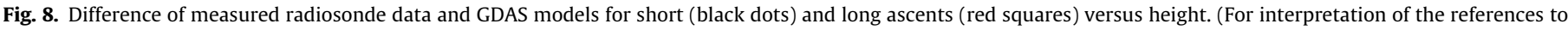
color in this figure legend, the reader is referred to the web version of this article.)

ger, the nMMM do not describe the conditions measured during the years 2009 and 2010 very well. In contrast, the GDAS data can represent the local conditions much better and the intrinsic uncertainty is consistently small. 
In the following, the non-perfect agreement between GDAS data and local sounding data below approximate $5 \mathrm{~km}$ a.s.l. is investigated in more detail. This effect close to ground could be caused by the nearby Andes and their influence on the climate above the array. GDAS was developed for global atmospheric predictions and therefore could be inadequate for very local atmospheric conditions. Under normal circumstances, the wind carries weather balloons launched at the Pampa Amarilla north-east or east with a horizontal displacement of about $100-150 \mathrm{~km}$ for ascents up to about $20 \mathrm{~km}$ a.s.l. In some cases however, the balloon just ascends with only a small horizontal drift in any direction, whereas the opposite extreme cases are horizontal displacements of more than
$200 \mathrm{~km}$. For this study, 25 short and localized ascents and 18 launches with very long balloon paths were selected manually. The differences between GDAS data and the measured data for both groups are shown in Fig. 8. The pressure data from GDAS do not describe these extreme conditions at the Auger Observatory as well as those seen under normal circumstances. For extremely short soundings, the local measurements reveal a high pressure area close to ground. This typically quite local effect is not reproduced by GDAS but for higher altitudes, the model data fit very well again. High pressure zones often indicate a stable atmospheric layering, so conditions change only on long-term scales. This could cause the good description of temperature profiles by GDAS. For
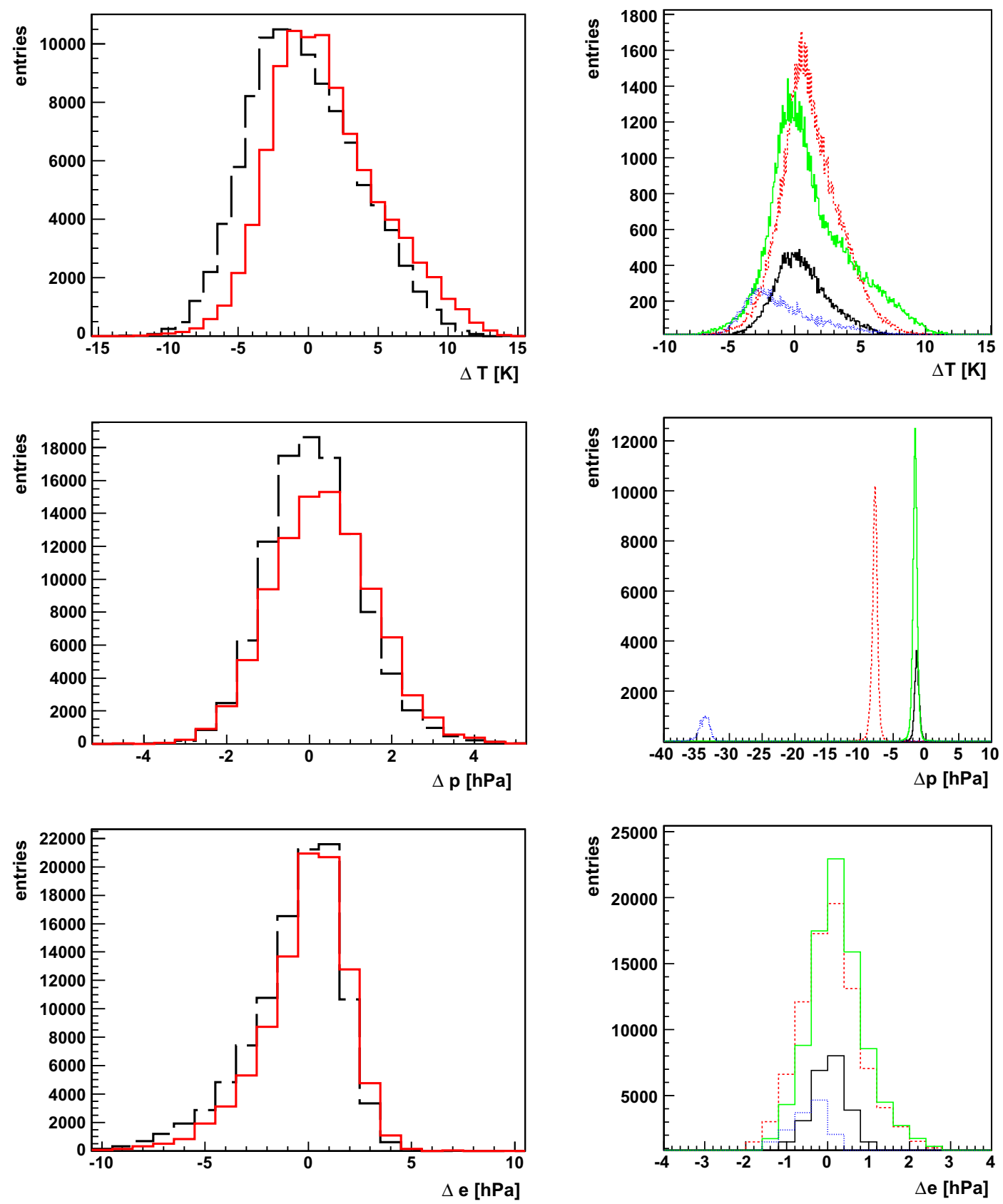

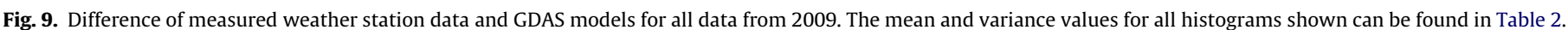

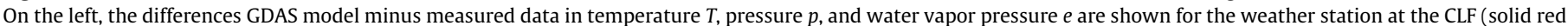

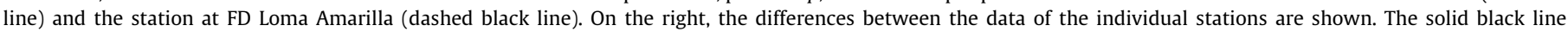

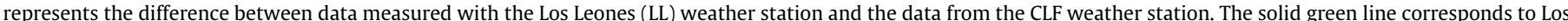

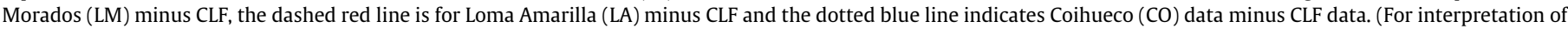
the references to color in this figure legend, the reader is referred to the web version of this article.) 
Table 2

Mean and variance values for the histograms shown in Fig. 9. Values for the pressure were not corrected for the height difference of the stations, but they are consistent with those differences. Applying the USStdA, the pressure difference due to the different altitudes of the weather stations are $-2.0 \mathrm{hPa}(\mathrm{LL}),-8.6 \mathrm{hPa}$ (LA), $-2.3 \mathrm{hPa}$ (LM), and $-32.8 \mathrm{hPa}(\mathrm{CO})$.

\begin{tabular}{|c|c|c|c|c|c|c|}
\hline & \multicolumn{3}{|l|}{ Mean } & \multicolumn{3}{|l|}{ RMS } \\
\hline & $T[\mathrm{~K}]$ & $p$ [hPa] & $e[\mathrm{hPa}]$ & $T[\mathrm{~K}]$ & $p[\mathrm{hPa}]$ & $e[\mathrm{hPa}]$ \\
\hline \multicolumn{7}{|c|}{$x_{\mathrm{WS}}-x_{\mathrm{GDAS}}$} \\
\hline CLF & 1.3 & 0.4 & -0.2 & 3.9 & 1.2 & 2.1 \\
\hline LA & -0.3 & 0.2 & -0.7 & 3.9 & 1.1 & 2.3 \\
\hline \multicolumn{7}{|c|}{$x_{\mathrm{WS}}-x_{\mathrm{CLF}}$} \\
\hline LL & 0.6 & -1.4 & -0.2 & 2.4 & 0.4 & 1.2 \\
\hline LA & 1.2 & -7.8 & 0.2 & 2.5 & 0.5 & 1.3 \\
\hline LM & 1.2 & -1.7 & 0.3 & 3.2 & 0.4 & 1.1 \\
\hline $\mathrm{CO}$ & -0.6 & -33.9 & -0.9 & 3.2 & 0.8 & 1.5 \\
\hline
\end{tabular}

the other extreme case, the launches with very long balloon paths, the local pressure data indicate a low pressure area, accompanied by significant winds. The conditions are dominated by turbulences, indicated by short-term and small-scale temperature variations. Thus, the GDAS model data do not fit the local measurements well. Overall, it is rather difficult to say if the topology of the Andes is the source of uncertainty or if it is also an effect of quite extreme weather conditions which might be induced by the structure of the Andes in the vicinity of the Pampa Amarilla. Moreover, both groups of investigated ascents are extremes and do not describe the usual conditions. Less than $20 \%$ of the ascents launched at the Pierre Auger Observatory fall into either category.
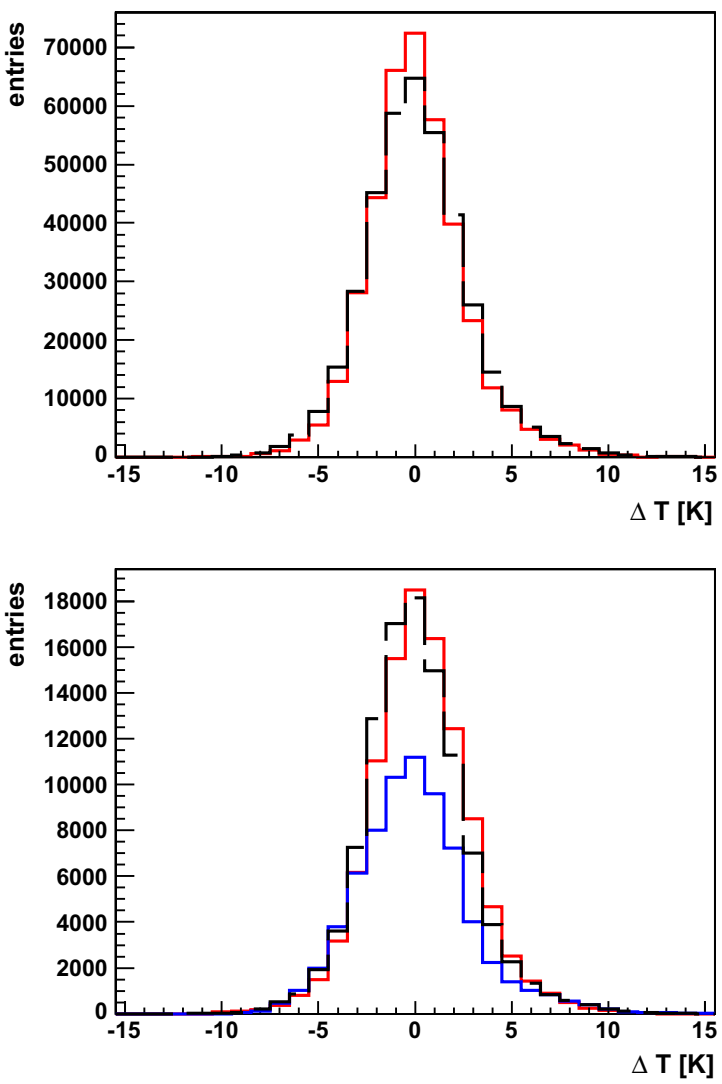

Finally, possible inconsistencies between local measurements and GDAS data close to the surface are investigated by using weather station data. While radiosondes may suffer from measurement uncertainties near the ground, the weather stations are specifically designed to continuously measure the ground values.

\subsection{GDAS vs. ground weather stations}

Five weather stations continuously monitor atmospheric values close to the ground, at about $2-4 \mathrm{~m}$ above surface level. Four are located at the FD stations, and one was set up near the center of the array at the Central Laser Facility (CLF). To make sure that the GDAS data describe the conditions at the ground reasonably well, the values provided by the GDAS data set are compared to all available weather station data. The GDAS data are interpolated at the height of the weather station.

In the histograms on the left of Fig. 9, the differences between measured weather station data and GDAS model data are shown for the weather stations close to the CLF and FD Loma Amarilla. All data measured in 2009 were used. Temperature, pressure, and water vapor pressure agree very well. Details of the histograms are listed in Table 2.

On the right side of Fig. 9, the differences of the data of the individual weather stations are shown. The CLF weather station is close to the middle of the array and was chosen as a reference. Values for the pressure were not corrected for the height difference of the stations, but they are consistent with the height differences of the stations. The mean and width of the CLF-LA distribution is very similar to the distributions on the left for the GDAS data. These two
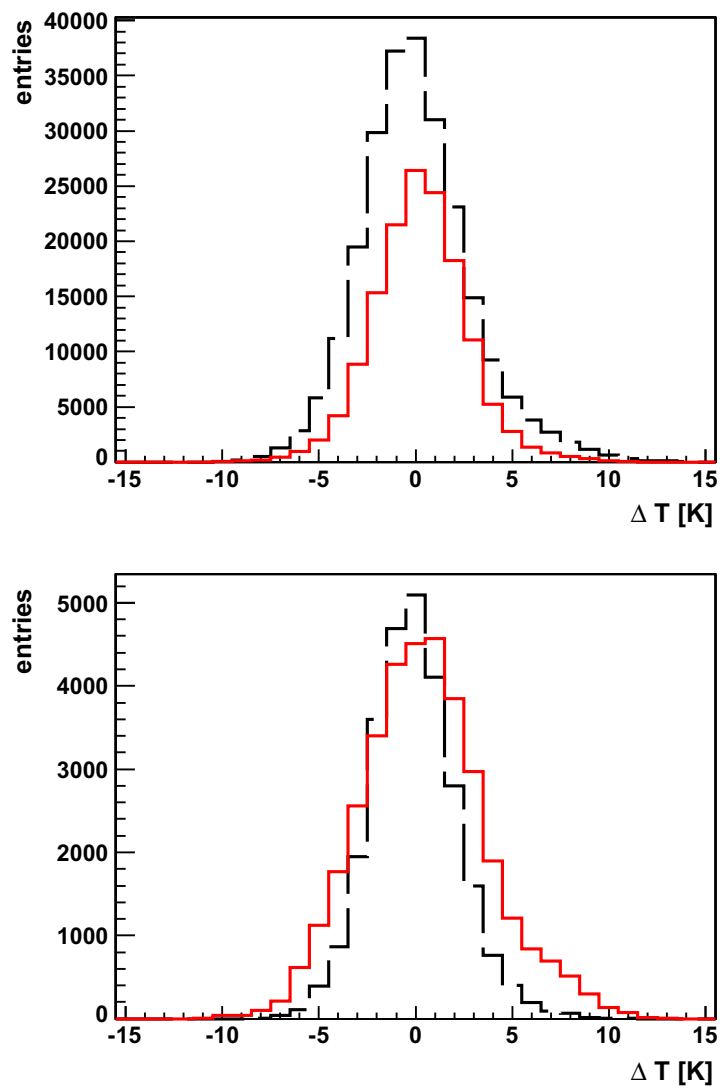

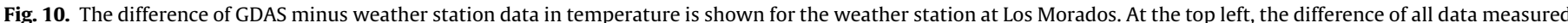

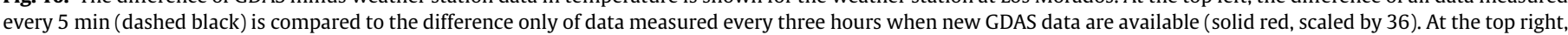

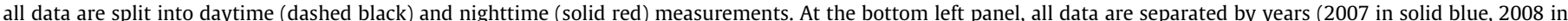

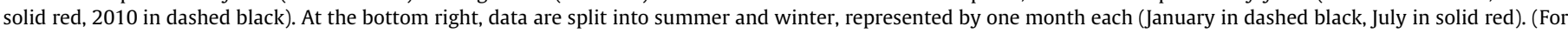
interpretation of the references to color in this figure legend, the reader is referred to the web version of this article.) 
histograms are expected to be similar because of the vicinity of the selected GDAS grid point and the FD building Loma Amarilla. Overall, the differences between the GDAS data and the weather station data are of the same order as the difference in data of two different weather stations. Only the difficult predictability of water vapor in the atmosphere close to ground can be seen again.

In Fig. 10, the differences in temperature for the weather station at Los Morados are analyzed in more detail. At the top left, all data measured every 5 min are compared with GDAS data. Additionally, only station data every three hours at which new GDAS data are available are shown, scaled by a factor of 36 . Both distributions show identical means of $0.1 \mathrm{~K}$, as well as similar RMS values of $2.7 \mathrm{~K}$ and $2.6 \mathrm{~K}$ for all data and for the 3-hourly data, respectively. The atmospheric parameters at the Observatory are very stable during 3-hour periods. At the top right, the data are split into day and night. Night is defined as the UTC hours between 0 and 10 . Therefore, with 14 compared to $10 \mathrm{~h}$ of data, the daytime distribution contains more data. For Los Morados, both distributions are close to each other, with a mean of $0.0 \mathrm{~K}$ and RMS of $2.9 \mathrm{~K}$ during the day and a mean of $0.2 \mathrm{~K}$ and RMS of $2.4 \mathrm{~K}$ at night. A small difference at night is noticeable, with the GDAS data giving higher temperatures than the weather station. The distributions for different years (bottom left) and different seasons (bottom right), represented by one month of summer and one from winter, show no distinct features or differences. Similar studies for pressure and water vapor pressure mostly yield similar results. Nevertheless, two deviations from this general pattern are discussed in the following.

In the left panel of Fig. 11, the temperature distributions for the station at Loma Amarilla are shown, split into daytime (solid red) and nighttime (dashed black) measurements. A clear separation of the two distributions is found. The mean of the distributions is $-1.6 \mathrm{~K}$ for daytime and $1.6 \mathrm{~K}$ for nighttime, resulting in a difference of $3.2 \mathrm{~K}$. This difference might be due to the local environment in which the weather station is placed. While some stations are far away from the FD buildings, others had to be mounted closer to or on top of other facilities. Thus, a standardized meteorological measurement cannot be guaranteed.

On the right of Fig. 11, the water vapor pressure for different seasons measured at the weather station at the CLF is shown. Clear differences are apparent. In austral winter (July), the water vapor pressure is very low, and the measurements agree better with GDAS data, the mean of the distribution is $0.8 \mathrm{hPa}$ with an RMS of $1.2 \mathrm{hPa}$. In summer (January), the deviations are largest, where the mean difference drops to $-1.4 \mathrm{hPa}$, and the RMS doubles to
$2.4 \mathrm{hPa}$. This indicates that the GDAS description of humidity is not perfect, underestimating the humidity in summer. However, the water vapor pressure calculation strongly depends on the temperature, so differences in temperature due to local effects of the surroundings of the station also affect this comparison.

Apart from the differences that were seen between radiosonde data and GDAS data near the surface in the previous section, the comparison of GDAS data with weather station data shows a very good agreement. We conclude that the GDAS data describe the conditions at the Pierre Auger Observatory very well. Because of their highly reliable availability and high frequency of data sets, GDAS data are a suitable replacement for local radio soundings and also for the local monthly models.

\subsection{GDAS vs. radio soundings at other locations}

In addition to the measurements in Argentina, similar radiosonde launches were performed in south-east Colorado, United States. 27 weather balloons were launched between September 2009 and December 2010 using identical radiosondes and equipment. The comparisons with the GDAS data for this location show differences of the same order as for the location of the Pierre Auger Observatory. This is remarkable because most of the global atmospheric models, in particular those developed in Europe and North America, typically describe the conditions at the northern hemisphere much better. This is due to the fact that atmospheric measurements in South America and in general at the southern hemisphere are sparse and accurate modeling of the atmosphere is predicated on real data.

Comparisons of GDAS data and radio soundings at further locations would go beyond the scope of this paper. Moreover, the radio soundings from south-east Colorado are independent data while other available data are from radio sounding databases which are part of the global meteorological network used for the creation of GDAS.

\section{Air shower reconstruction}

To study the effects caused by using the GDAS data, all air shower data from the Auger Observatory collected between June 1, 2005 and end of 2010 are used in a reconstruction analysis using the Offline software framework of the Pierre Auger Observatory [24]. The change of atmosphere description will mainly affect the reconstruction of the fluorescence data, c.f. Section 2. Therefore,
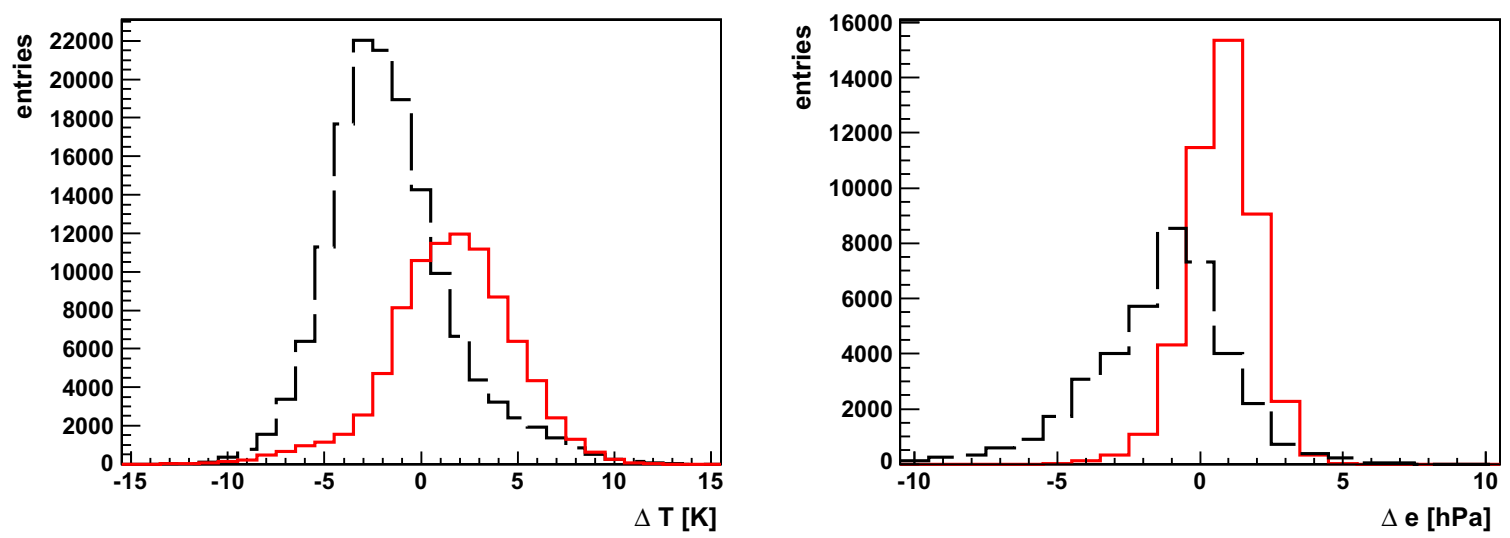

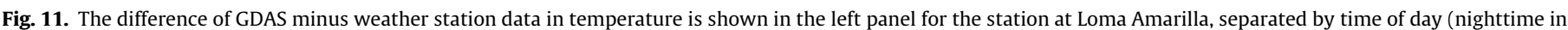

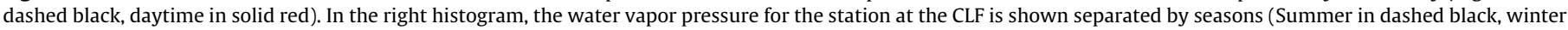
in solid red). (For interpretation of the references to color in this figure legend, the reader is referred to the web version of this article.) 
we concentrate on this part in the following. It is known that varying atmospheric conditions alter the fluorescence light production and transmission [3]. The transmittance of the actual atmosphere is regularly measured during FD shifts and made available for air shower reconstructions via databases. The light production has to be calculated analytically during the reconstruction procedure. Its strong atmosphere-dependence as described in Section 2 is applied in the air shower reconstruction analysis.

\subsection{Data reconstruction}

The following analysis is based on three sets of reconstructions. The first set, FY, is the until recently standard reconstruction of the Pierre Auger Observatory. The fluorescence yield is calculated with its atmosphere-dependence as described in [25], along with the monthly mean profiles (nMMM) obtained for the site of the Auger Observatory. For the second set, $\mathrm{FY}_{\text {mod, }}$, all currently known atmospheric effects in the fluorescence calculation are taken into account. Together with the standard atmosphere-dependence, the temperature-dependent collisional cross sections and humidity quenching are included ([7] and references therein). Parameterizations for these two effects are taken from AIRFLY [8] and later conference contributions by the AIRFLY collaboration. Again, the nMMM are used. The third set, $F Y_{\text {mod }}^{\text {GDAS }}$, also explores the efficiency of the full atmosphere-dependent fluorescence description, but here the atmospheric nMMM are exchanged with the new 3hourly GDAS data.

Comparing the reconstruction sets with each other, the variation of the reconstructed primary energy $E$ of air showers and the position of shower maximum $X_{\max }$ can be determined, see Fig. 12. In the two upper figures, the binned difference of $E$ and $X_{\max }$ is displayed, and the dependences on energy and month of these differences are shown in the figures in the middle and bottom, respectively.

Using GDAS data for the reconstruction instead of nMMM affects the reconstructed primary energy only slightly. The mean of the difference $F Y_{\text {mod }}^{G D A S}$ minus $F Y_{\text {mod }}$ is $0.4 \%$ with an RMS of $1.4 \%$ (Fig. 12, top left, solid black line). For the reconstructed $X_{\max }$, only a small shift of $-1.1 \mathrm{~g} \mathrm{~cm}^{-2}$ is found with an RMS of $6.0 \mathrm{~g} \mathrm{~cm}^{-2}$ (Fig. 12, top right, solid black line). However, comparing the full
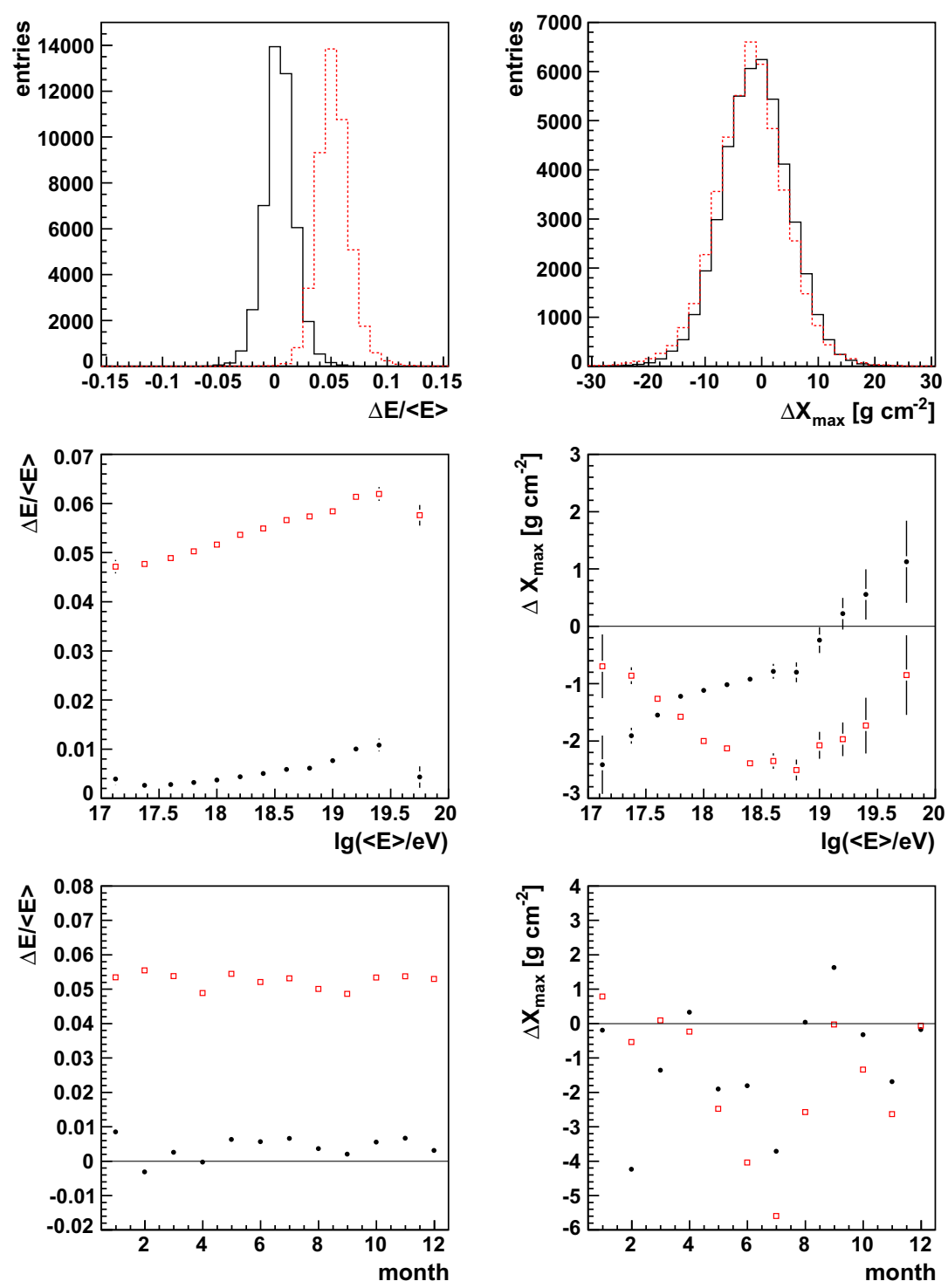

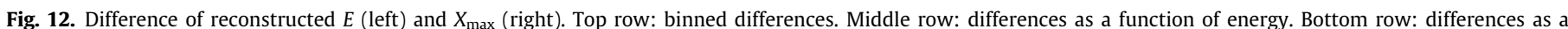

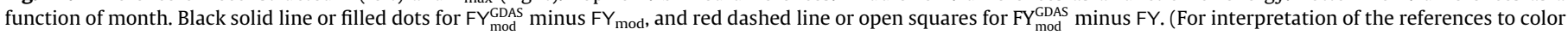
in this figure legend, the reader is referred to the web version of this article.) 
atmosphere-dependent reconstruction $F Y_{\bmod }^{G D A S}$ with the unmodified reconstruction $\mathrm{FY}$, a clear shift in $E$ can be seen. An increase in $E$ by $5.2 \%$ (RMS $1.5 \%$ ) and a decrease of $X_{\max }$ by $-1.9 \mathrm{~g} \mathrm{~cm}^{-2}$ (RMS $6.3 \mathrm{~g} \mathrm{~cm}^{-2}$ ) is found. These modified fluorescence settings are now used in the reconstruction of the Pierre Auger Observatory, in conjunction with other improvements to the procedure, see [26].

The difference in reconstructed $E$ vs. mean $E$ (Fig. 12, middle left) reveals a small energy dependence, increasing towards higher energies. The description of atmospheric conditions close to the ground is very difficult in monthly mean profiles since the fluctuations in temperature and humidity are larger in the lower levels of the atmosphere (below $4 \mathrm{~km}$ ) than in the upper layers. Consequently, a more precise description of actual atmospheric conditions with GDAS will alter the energy reconstruction compared with nMMM-based reconstructions for air showers that penetrate deeply into the atmosphere, usually high-energy events. The full atmosphere-dependent fluorescence calculation alters the light yield for conditions with very low temperatures, corresponding to higher altitudes. The energy dependence of the $X_{\max }$ differences is a combined effect of slightly changed humidity conditions close to ground and temperature conditions higher up in the atmosphere together with the full atmosphere-dependent fluorescence calculation (Fig. 12, middle right).

The difference in energy is quite uniform throughout the year, see Fig. 12, bottom left. For switching on GDAS instead of nMMM (black dots), it is confirmed that GDAS describes the conditions at the Auger Observatory very well, as good as the nMMM. Switching on the full atmosphere-dependent fluorescence calculation does not show a monthly dependence because the overall integral of the longitudinal light profiles is hardly changed, see, e.g. [27]. Only the modification of the shape of the longitudinal light profile causes a small monthly dependence of $X_{\max }$ (Fig. 12, bottom right).

In the following, some systematics caused by the particular shower geometry are studied. In the first set of figures (Fig. 13, top row), the difference in $E$ and $X_{\max }$ vs. zenith angle of the shower $\theta$ is displayed. The energy variation is quite uniform around the mean value up to about $60^{\circ}$. Only more inclined showers show a stronger shift of reconstructed energy for the modified fluorescence yield calculation. Concerning the position of shower maximum, a dependence on zenith angle can be seen above $30^{\circ}$.

Shown in the bottom row of Fig. 13, the $E$ and $X_{\max }$ dependence on geometrical height of shower maximum gives a more pronounced view of the atmospheric conditions in combination with the atmosphere-dependent fluorescence description. Showers reaching their maximum at an average altitude between 3 and $7 \mathrm{~km}$ show the mean $E$ difference as expected from Fig. 12, top left. However, showers with very shallow or very deep positions of shower maximum are reconstructed with a $7-8 \%$ higher primary energy compared with that using the standard fluorescence calculation. The reconstructed $X_{\max }$ follows the expectations according to the study shown in [27].

\subsection{Impact on shower reconstruction uncertainties}

To study the effect that the GDAS data have on the uncertainties of air shower reconstructions, air showers induced by protons and iron nuclei are simulated using the CONEX shower generator [28] with the QGSJETII hadronic interaction model [29] for shower energies between $10^{17.5} \mathrm{eV}$ and $10^{20} \mathrm{eV}$. The fluorescence light is generated including temperature-dependent collisional cross sections and vapor quenching. The time stamps of the air shower events correspond to the times of 109 radio soundings between August 2002 and December 2008 so that actual atmospheric profiles can be used in the simulation. All 109 launches were performed at night during cloud-free conditions. After the atmospheric transmission, the detector optics and electronics are simulated. The resulting data are then reconstructed using the radiosonde data, as well as the GDAS data.
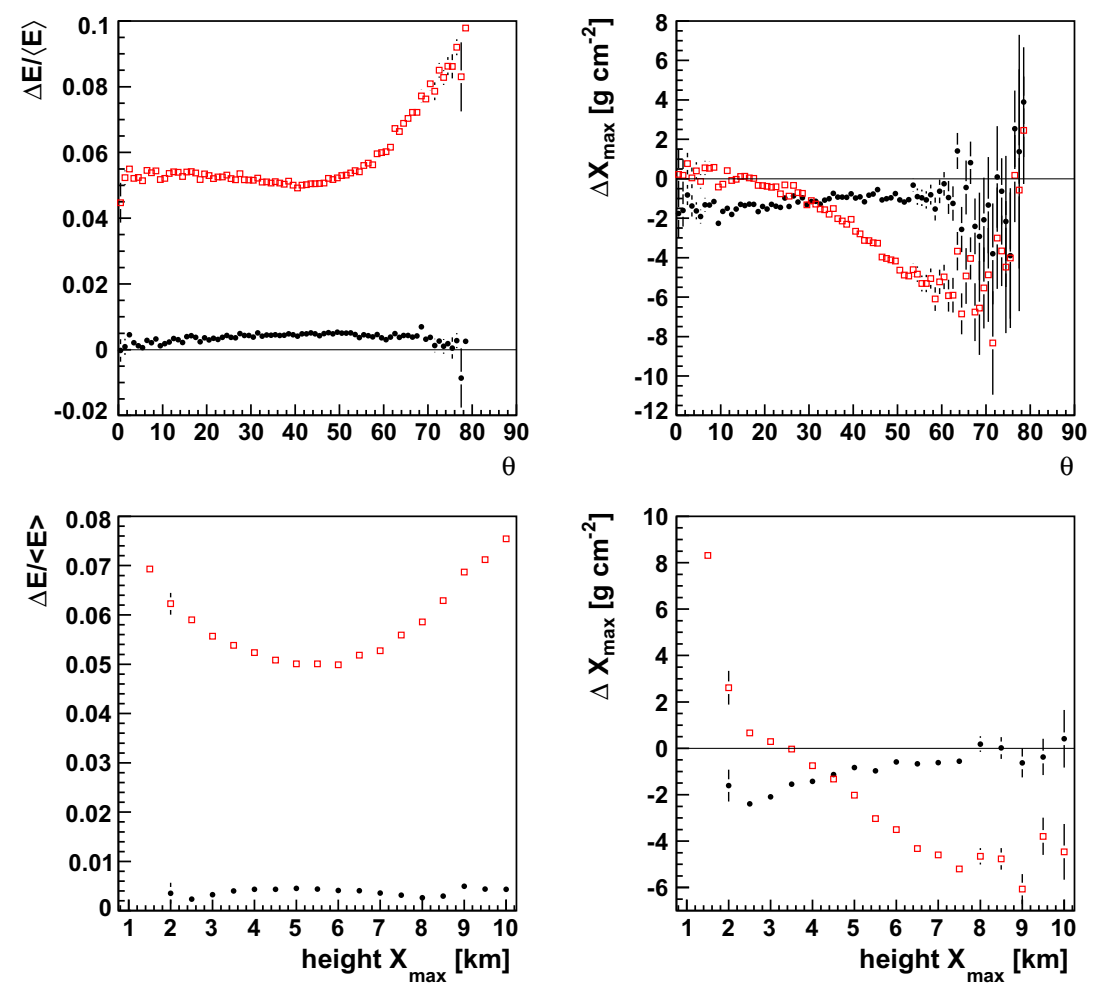

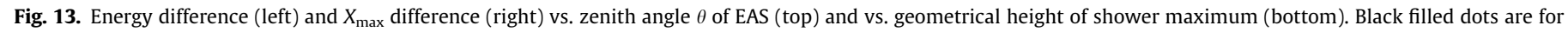
$F Y_{\text {mod }}^{G D A S}$ minus $F Y_{\text {mod, }}$, and red open squares for $F Y_{\bmod }^{G D A S}$ minus $F Y$. 

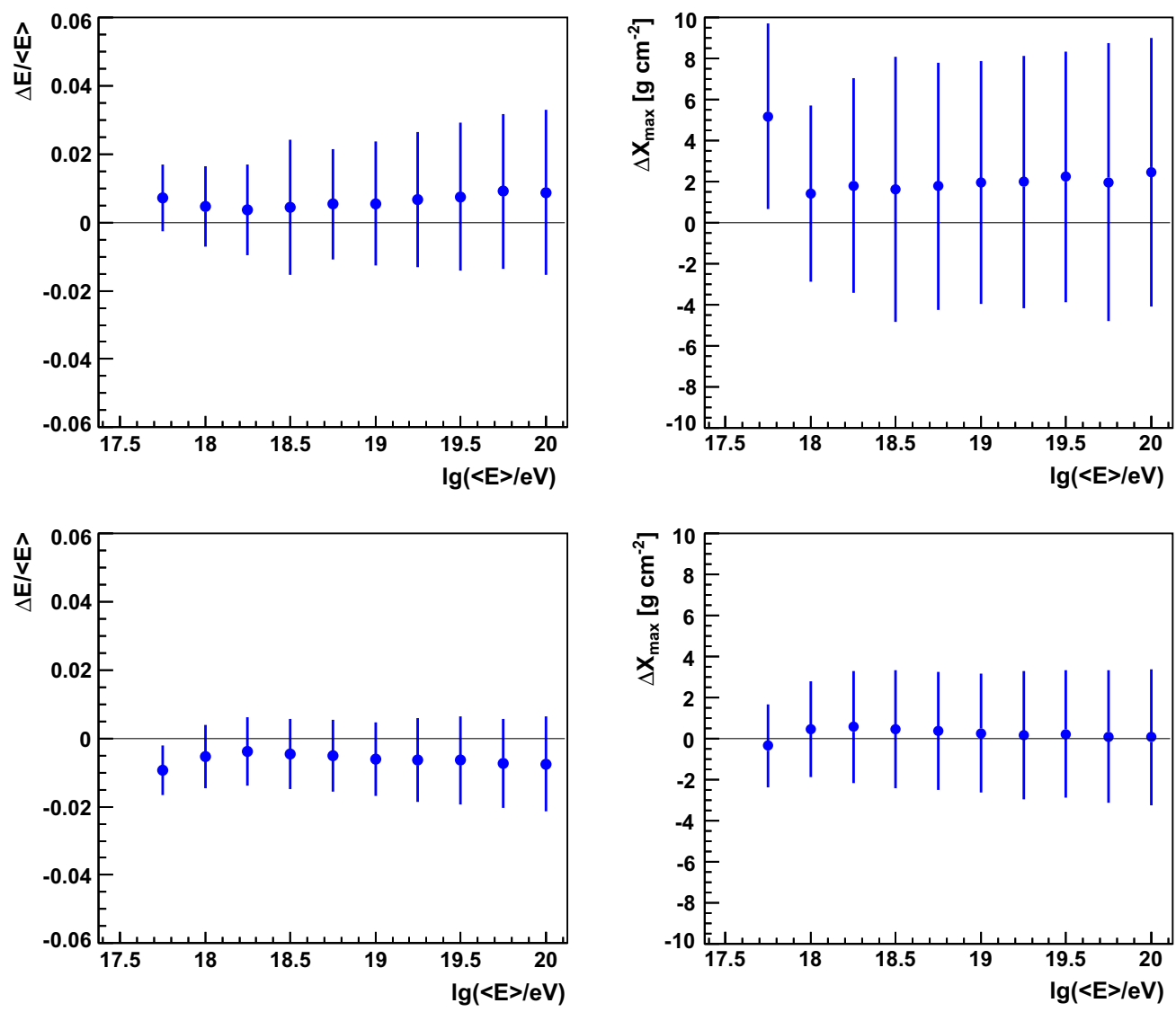

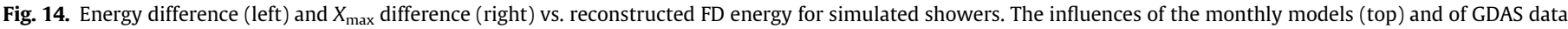
(bottom) are shown. Error bars denote the RMS spread.

A basic set of quality cuts is applied. The shower maximum has to be in the observed part of the track, and the uncertainty in reconstructed energy and $X_{\max }$ must be below $20 \%$ and $40 \mathrm{~g} \mathrm{~cm}^{-2}$, respectively. Also, the Gaisser-Hillas profile fit has to have a given quality, $\chi^{2} / \mathrm{Ndf}<2$, and the fraction of Cherenkov light from the shower observed by the telescopes must be less than $50 \%$. After applying all cuts, the values of energy and $X_{\max }$ from the reconstructions with different atmospheric description are compared. The differences in these reconstructions yield the uncertainties that are introduced by the use of the GDAS data instead of the actual atmospheric profile.

The same study has been performed to determine the uncertainties from the nMMM [30]. There, the systematic error is less than $1 \%$ in energy and less than $2 \mathrm{~g} \mathrm{~cm}^{-2}$ in $X_{\max }$. In the energy range from $10^{17.5} \mathrm{eV}$ to $10^{20} \mathrm{eV}$, random energy-dependent reconstruction uncertainties of $\pm 1 \%$ and $\pm 5 \mathrm{~g} \mathrm{~cm}^{-2}$ for low energies up to $\pm 2 \%$ and $\pm 7 \mathrm{~g} \mathrm{~cm}^{-2}$ for high energies were found. In the course of our new study, we compute the same uncertainties due to the nMMM again. The main difference between the current analysis and the previous one is the implementation of the temperaturedependent collisional cross sections and the humidity quenching in the calculation of the fluorescence yield.

In Fig. 14, both results of the new study are shown, the uncertainties due to nMMM and those due to GDAS. A deviation from zero indicates a systematic error, and the error bars denote the RMS spread of all simulated events and are a measure of the reconstruction uncertainty due to this atmospheric parameterization. In the top panels, the influence of the nMMM compared to the real atmospheric parameters from radiosondes is shown. The results for the systematic shifts are in agreement with the previous study [30], with only the systematic shift for $X_{\max }$ in the lowest energy bin being higher. The RMS spread in energy is $\pm 0.9 \%$ for low energies and up to $\pm 2.4 \%$ for high energies. In $X_{\max }$, the RMS is between $\pm 4 \mathrm{~g} \mathrm{~cm}^{-2}$ for low energies and $\pm 6.5 \mathrm{~g} \mathrm{~cm}^{-2}$ for high energies.

The influence on the reconstruction due to GDAS data is shown in the bottom part of Fig. 14. The systematic shifts in energy are of the same order, below $1 \%$, but of opposite sign. The shifts in $X_{\max }$ are much smaller than for the nMMM, less than $0.5 \mathrm{~g} \mathrm{~cm}^{-2}$. The RMS spread is also considerably smaller, $\pm 0.9 \%$ and $\pm 2 \mathrm{~g} \mathrm{~cm}^{-2}$ for low energies, and $\pm 1.3 \%$ and $\pm 3.5 \mathrm{~g} \mathrm{~cm}^{-2}$ for high energies. The energy uncertainty at low energies is comparable to the uncertainty introduced by the nMMM, but at high energies the uncertainty is reduced by almost $50 \%$. For $X_{\max }$, the uncertainties in all energy bins are halved.

This study of the reconstruction uncertainties further demonstrates the advantages of GDAS data over the monthly mean profiles.

\section{Conclusion}

The reconstructions of air showers measured at the Pierre Auger Observatory have used a set of monthly mean profiles as the standard atmospheric description until recently. These profiles are averages from meteorological radio soundings performed at the site of the observatory over several years. The mean profiles describe the local conditions reasonably well, but cannot describe short-term variations in the atmosphere. Because of the large burden radio soundings impose on the collaboration, and their ambiguous duration of validity, data from the Global Data Assimilation System (GDAS) are a welcome substitute for atmospheric descriptions. GDAS data rely on established meteorological models and have an excellent time resolution of $3 \mathrm{~h}$. 
A direct comparison of GDAS data for the site of the Auger Observatory with local atmospheric measurements validates the adequate accuracy of GDAS data with respect to horizontal and vertical as well as temporal resolution. The suitable online publication of these data by NCEP allows for an easy and timely updating of atmospheric databases used at the Pierre Auger Observatory.

With an air shower reconstruction analysis, the applicability of GDAS data to air shower analyses can be confirmed along with an improved accuracy with respect to atmospheric conditions. Also, the value of using an atmosphere-dependent fluorescence description has been demonstrated. Using simulated air showers, we show that the GDAS data significantly reduce the systematic errors and overall uncertainties in air shower reconstructions.

Because of the results discussed in this study, the standard air shower analyses of the Pierre Auger Observatory are now applying atmospheric data from GDAS and the fluorescence description FY ${ }_{\text {mod }}^{\text {GDAS }}$ as described in Section 6.

\section{Acknowledgments}

The successful installation, commissioning and operation of the Pierre Auger Observatory would not have been possible without the strong commitment and effort from the technical and administrative staff in Malargüe.

We are very grateful to the following agencies and organizations for financial support: Comisión Nacional de Energía Atómica, Fundación Antorchas, Gobierno De La Provincia de Mendoza, Municipalidad de Malargüe, NDM Holdings and Valle Las Leñas, in gratitude for their continuing cooperation over land access, Argentina; the Australian Research Council; Conselho Nacional de Desenvolvimento Científico e Tecnológico (CNPq), Financiadora de Estudos e Projetos (FINEP), Fundação de Amparo à Pesquisa do Estado de Rio de Janeiro (FAPERJ), Fundação de Amparo à Pesquisa do Estado de São Paulo (FAPESP), Ministério de Ciência e Tecnologia (MCT), Brazil; AVCR, AV0Z10100502 and AV0Z10100522, GAAV KJB300100801 and KJB100100904, MSMT-CR LA08016, LC527, 1M06002, and MSM0021620859, Czech Republic; Centre de Calcul IN2P3/CNRS, Centre National de la Recherche Scientifique (CNRS), Conseil Régional Ile-de-France, Département Physique Nucléaire et Corpusculaire (PNC-IN2P3/CNRS), Département Sciences de l'Univers (SDU-INSU/CNRS), France; Bundesministerium für Bildung und Forschung (BMBF), Deutsche Forschungsgemeinschaft (DFG), Finanzministerium Baden-Württemberg, HelmholtzGemeinschaft Deutscher Forschungszentren (HGF), Ministerium für Wissenschaft und Forschung, Nordrhein-Westfalen, Ministerium für Wissenschaft, Forschung und Kunst, Baden-Württemberg, Germany; Istituto Nazionale di Fisica Nucleare (INFN), Istituto Nazionale di Astrofisica (INAF), Ministero dell'Istruzione, dell'Università e della Ricerca (MIUR), Gran Sasso Center for Astroparticle Physics (CFA), Italy; Consejo Nacional de Ciencia y Tecnología (CONACYT), Mexico; Ministerie van Onderwijs, Cultuur en Wetenschap, Nederlandse Organisatie voor Wetenschappelijk Onderzoek (NWO), Stichting voor Fundamenteel Onderzoek der Materie (FOM), Netherlands; Ministry of Science and Higher Education, Grant Nos. 1 P03 D 01430 and N N202 207238, Poland; Fundação para a Ciência e a Tecnologia, Portugal; Ministry for Higher Education, Science, and Technology, Slovenian Research Agency, Slovenia; Comunidad de Madrid, Consejería de Educación de la Comunidad de Castilla La Mancha, FEDER funds, Ministerio de Ciencia e Innovación and Consolider-Ingenio 2010 (CPAN), Generalitat Valenciana, Junta de Andalucía, Xunta de Galicia, Spain; Science and Technology Facilities Council, United Kingdom; Department of Energy, Contract Nos. DE-AC02-07CH11359, DE-FR02-
04ER41300, National Science Foundation, Grant No. 0969400, The Grainger Foundation USA; ALFA-EC/ HELEN, European Union 6th Framework Program, Grant No. MEIF-CT-2005-025057, European Union 7th Framework Program, Grant No. PIEF-GA-2008-220240, and UNESCO.

\section{References}

[1] J. Abraham et al., Properties and performance of the prototype instrument for the Pierre Auger Observatory, Nucl. Instrum. Methods A523 (2004) 50-95.

[2] J. Abraham et al., The fluorescence detector of the Pierre Auger Observatory, Nucl. Instrum. Methods A620 (2010) 227-251, doi:10.1016/ j.nima.2010.04.023. <arxiv:0907.4282>.

[3] J. Abraham et al., A study of the effect of molecular and aerosol conditions in the atmosphere on air fluorescence measurements at the Pierre Auger Observatory, APh 33 (2010) 108-129. <arXiv:1002.0366>.

[4] J. Abraham et al., Atmospheric effects on extensive air showers observed with the surface detector of the Pierre Auger Observatory, APh 32 (2009) 89-99. <arXiv:0906.5497>.

[5] K. Louedec, The Pierre Auger Collaboration, Atmospheric monitoring at the pierre auger observatory - status and update, in: Proc. 32nd ICRC, Beijing, China, 2011, <arXiv:1107.4806>.

[6] B. Keilhauer, The Pierre Auger Collaboration, The balloon-the-shower programme of the Pierre Auger Observatory, Astrophys. Space Sci. Trans. 6 (2010) 27-30.

[7] F. Arqueros, J. Hörandel, B. Keilhauer, Air fluorescence relevant for cosmic-ray detection - summary of the 5th fluorescence workshop, El Escorial 2007, Nucl. Instrum. Methods A597 (2008) 1-22. <arXiv:0807.3760>.

[8] M. Ave et al., Temperature and humidity dependence of air fluorescence yield measured by AIRFLY, Nucl. Instrum. Methods A597 (2008) 50-54.

[9] M. Boháčová, The AIRFLY Collaboration, Temperature and humidity dependence of air fluorescence yield, in: 6th Air Fluorescence Workshop, L'Aquila, Italy, 2009.

[10] M. Nagano et al., New measurement on photon yields from air and the application to the energy estimation of primary cosmic rays, APh 22 (2004) 235-248.

[11] J.C. Owens et al., Temperature and composition, Appl. Opt. 6 (1967) 51-59.

[12] P.E. Ciddor et al., Refractive index of air: 3. The roles of $\mathrm{CO}_{2}, \mathrm{H}_{2} \mathrm{O}$, and refractivity virials, Appl. Opt. 41 (2002) 2292-2298.

[13] K. Birch, M. Downs, An updated Edlén equation for the refractive index of air, Metrologia 30 (1993) 155-162.

[14] C. Tomasi et al., Improved algorithm for calculations of Rayleigh-scattering optical depth in standard atmospheres, Appl. Opt. 44 (2005) 3320-3341.

[15] B. Keilhauer et al., The Pierre Auger Collaboration, Rapid atmospheric monitoring after the detection of high-energy showers at the Pierre Auger Observatory, in: Proc. 31st ICRC, Łódź, Poland, 2009, <arXiv:0906.2358>.

[16] P. Müller, H. von Storch, Computer Modeling in Atmospheric and Oceanic Sciences, Springer Verlag, 2004.

[17] W. Wergen, Datenassimilation - ein Überblick, Promet 27 (3/4) (2002) 142149 (in German).

[18] NOAA Air Resources Laboratory (ARL), Global Data Assimilation System (GDAS1) Archive Information, Tech. rep., <http://ready.arl.noaa.gov/gdas1. php>, 2004.

[19] National Aeronautics and Space Administration (NASA), US Standard Atmosphere 1976, NASA-TM-X-74335, 1976.

[20] National Centers for Environmental Prediction (NCEP), GFS/G DAS Changes Since 1991, Tech. rep., <http://www.emc.ncep.noaa.gov/gmb/STATS/html/ model changes.html>, 2010.

[21] B. Stunder, NOAA Air Resources Laboratory, private communication, 2011.

[22] M. Mahoney, A Discussion of Various Measures of Altitude, Tech. rep., <http:// mtp.mjmahoney.net/www/notes/altitude/altitude.html>, 2008.

[23] B. Bodhaine et al., On Rayleigh optical depth calculations, J. Atmos. Ocean. Tech. 16 (1999) 1854-1861.

[24] S. Argirò et al., The offline software framework of the Pierre Auger Observatory, Nucl. Instrum. Methods A580 (2007) 1485-1496. <arXiv:0707.1652>.

[25] M. Ave et al., The AIRFLY Collaboration, Measurement of the pressure dependence of air fluorescence emission induced by electrons, APh 28 (2007) 41-57.

[26] R. Pesce et al., The Pierre Auger Collaboration, Energy calibration of data recorded with the surface detectors of the Pierre Auger Observatory: an update, in: Proc. 32nd ICRC, Beijing, China, 2011, <arXiv:1107.4809>.

[27] B. Keilhauer, J. Blümer, R. Engel, H. Klages, Altitude dependence of fluorescence light emission by extensive air showers, Nucl. Instrum. Methods A597 (2008) 99-104. <arXiv:0801.4200>.

[28] T. Bergmann et al., One-dimensional hybrid approach to extensive air shower simulation, APh 26 (2007) 420-432.

[29] S. Ostapchenko, QGSJET-II: towards reliable description of very high energy hadronic interactions, Nucl. Phys. Proc. Suppl. 151 (2006) 143-146.

[30] B. Keilhauer, M. Unger, Fluorescence emission induced by extensive air showers in dependence on atmospheric conditions, in: Proc. 31st ICRC, Łódź, Poland, 2009, <arXiv:0906.5487>. 